\title{
MEASURE-THEORETIC UNIFORMITY IN RECURSION THEORY AND SET THEORY
}

\author{
BY \\ GERALD E. SACKS $\left({ }^{1}\right)$
}

1. Introduction. The notion of measure-theoretic uniformity was presented in [23], [24]. In this paper the notion is first utilized in an arithmetic setting and then extended to hyperarithmetic theory and set theory. The recursion-theoretic concepts touched on are: the arithmetic, hyperarithmetic and analytic hierarchies of Kleene [12]; $\omega$-models of the hyperarithmetic comprehension axiom of Kreisel [16]; recursive ordinals [11]; and hyperdegrees [13]. One of the recursion-theoretic results we prove is: if $P(X)$ is a $\Pi_{1}^{1}$ predicate with free set variable $X$ and the set $\hat{X} P(X)$ has positive Lebesgue measure, then $P(A)$ holds for some hyperarithmetic set $A$. A corollary of this result is: the set of all $X$ such that the ordinals recursive in $X$ coincide with the recursive ordinals has Lebesgue measure 1 (see footnote $\left(^{9}\right.$ ), below). In the area of set theory we are largely concerned with showing how relative consistency results follow in a natural manner from the notion of measure-theoretic uniformity. We develop Cohen's independence results [2] as well as a result of Solovay [29], [30] on the extendability of Lebesgue measure to all sets of reals. Solovay makes use of Cohen's forcing method, but he ingeniously replaces Cohen's finite forcing conditions by closed sets of positive measure. He shows: if ZF (Zermelo-Fraenkel set theory) is consistent, then ZF + "there exists a translationinvariant, countably additive extension of Lebesque measure defined on all sets of reals" + "the dependent axiom of choice" is consistent $\left({ }^{2}\right)$. We reprove Solovay's theorem with emphasis on the notion of measure-theoretic uniformity and with the help of some elementary properties of uniformly distributed, independent random variables; however, the fine details of our argument are not substantially different from those originated by Solovay. $\S 4$ of this paper, which deals solely with set theory, can be read independently of $\S \S 2$ and 3, but it is intended to be read as a natural continuation of the earlier sections.

Received by the editors March 13, 1968 and, in revised form, August 1, 1968.

(1) The preparation of this paper was partially supported by U.S. Army Contract ARO-D373 and by NSF grant GP-6982. The author wishes to thank Professor A. Nerode for many helpful conversations on uniformity and definability, and Professor D. Lacombe for enabling the author to present the paper in a series of lectures [24] at the University of Paris in November, 1966.

$\left.{ }^{2}\right)$ R. B. Jensen has shown that the dependent axiom of choice is stronger than the countable axiom of choice. Solovay has also shown: if $\mathrm{ZF}+$ "there exists an inaccessible cardinal" is consistent, then so is $\mathrm{ZF}+$ "the dependent axiom of choice" + "all sets of reals are Lebesque measurable". 
Our interest in applying measure-theoretic ideas to questions of mathematical logic began with the reading of Spector's measure-theoretic construction of two incomparable hyperdegrees [32]. Spector showed, using Fubini's Theorem and the measurability of analytic sets, that the set of all pairs $(X, Y)$ such that $X$ and $Y$ are hyperarithmetically incomparable has measure 1 . Shoenfield extended Spector's argument to show there exists an uncountable set of pairwise incomparable hyperdegrees. Feferman [3] obtained two incomparable hyperdegrees by means of a forcing argument, but Spector's proof, although less constructive than Feferman's, is much shorter. In [22] we showed: if $X$ is not recursive, then the set of all $Y$ such that the Turing-degree of $Y$ is incomparable with that of $X$ has measure 1 . In $\S 3$ we show: if $X$ is not hyperarithmetic, then the set of all $Y$ such that the hyperdegree of $Y$ is incomparable with that of $X$ has measure 1 . There is a significant conceptual difference between these last two results. The proof of the former of the two relies on the countable additivity of Lebesgue measure and on the following fact: if $X$ is Turing-reducible to (i.e., recursive in) $Y$, then $X$ is reducible to $Y$ by means of one of countably many reduction procedures whose nature does not depend on $Y$ (i.e., $\left.X=\{e\}^{Y}\right)$. In contrast, if $X$ is hyperarithmetic in $Y$, then $X$ is reducible to $Y$ by means of a hyperarithmetic reduction procedure whose nature depends on the ordinals recursive in $Y$. Since the ordinals recursive in $Y$ can be arbitrarily large countable ordinals, the proof of the latter of the above two results has to take into account uncountably many hyperarithmetic reduction procedures. This difficulty is overcome in $\$ 3$ by showing that if one ignores a set of $Y$ 's of measure 0 , then one can assume the ordinals recursive in $Y$ are the recursive ordinals.

It seems impractical at this point to give a general and formal account of the notion of measure-theoretic uniformity. Instead, we give a variety of examples and applications in the belief that the notion is simple enough to abstract from the examples. Let $N$ be the natural numbers. We put the usual probability measure on $2^{N}$ as follows. Let $m$ be the unbiased measure for $2=\{0,1\}: m(\{0,1\})=1, m(\{0\})$ $=m(\{1\})=\frac{1}{2}$, and $m(\varnothing)=0$, where $\varnothing$ denotes the empty set. Let $\mu$ be the product measure induced on $2^{N}$ by $N$. Of course $\mu$ is merely the product-space version of Lebesgue measure on the unit interval $[0,1]$ of the reals $[10$, p. 157]. What we need of measure theory can be found in Halmos [10].

Let $R(T, x, y)$ be a recursive predicate [12] of the set variable $T$ and the natural number variables $x$ and $y$. Thus the truth-value of $R(T, x, y)$ for any actual choice of $T \in 2^{N}$ and $x, y \in N$ is determined by finitely much information about the membership of $T$ and the values of $x$ and $y$. A familiar uniformity can be put as follows:

$$
(T)[(x)(E y) R(T, x, y) \rightarrow(E f)(f \text { recursive in } T \&(x) R(T, x, f(x)))] .
$$

(In fact, $f(x) \cong \mu y R(T, x, y)$.) Another way of expressing this uniformity is: for each $T$, if the predicate $(x)(E y) R(T, x, y)$ has a Skolem function, then it has a Skolem function recursive in $T$. Thus in our study of the predicate $(x)(E y) R(T, x, y)$, 
we can reduce our attention from arbitrary Skolem functions to Skolem functions recursive in $T$. It is now quite natural to ask: can we reduce our attention still further to recursive Skolem functions? The answer is yes, if we ignore a set of $T$ 's of measure 0 , and if we replace Skolem functions by bounding functions: $f$ is a bounding function for $(x)(E y) R(T, x, y)$ if $(x)(E y)_{y \leqq f(x)} R(T, x, y)$. The existence of a bounding function is equivalent to the existence of a Skolem function.

Proposition 1.1. Let $R(T, x, y)$ be recursive. If the set of all $T$ such that $(x)(E y) R(T, x, y)$ has measure 1 , then the set of all $T$ such that $(E f)[f$ recursive \& $\left.(x)(E y)_{y \leqq f(x)} R(T, x, y)\right]$ also has measure 1 .

Proof. It is enough to show: for each rational $\delta>0$, there exists a recursive $f$ such that $\left\{T \mid(x)(E y)_{y \leqq f(x)} R(T, x, y)\right\}$ has measure at least $1-\delta$. A nonempty, basic open subset of $2^{N}$ consists of all $T$ satisfying a consistent conjunction of finitely many atomic conditions of the form $m \in T$ or their negations. The measure of a nonempty, basic open set is $2^{-n}$, where $n$ is the number of distinct atomic conditions that define the basic open set. Thus $\mu(\{T \mid m \in T \& m+1 \notin T\})=1 / 4$. Since $R$ is recursive, there exists a recursive function $b_{i}^{m, n}$ such that for each $m$ and $n$, $b_{i}^{m, n}$ is a basic open subset of $2^{N}$, and such that for all $T$,

$$
(m)(n)\left[R(T, m, n) \leftrightarrow(E i)\left(T \in b_{i}^{m, n}\right)\right] .
$$

For each $m$, let

$$
f(m)=\mu t\left[\mu\left(\bigcup\left\{b_{i}^{m, n} \mid i \leqq t \& n \leqq t\right\}\right) \geqq 1-\delta / 2^{m+1}\right] .
$$

For each $m, f(m)$ is defined because $\mu\left(\bigcup\left\{b_{i}^{m, n} \mid i \geqq 0 \& n \geqq 0\right\}\right)=1$. (Read " $\mu t$ " as "the least $t$ such that".)

A stronger and more attractive proposition than 1.1, and one closer to the idea of measure-theoretic uniformity would be: the set of all $T$ such that

$$
(x)(E y) R(T, x, y) \rightarrow(E f)\left[f \text { recursive } \&(x)(E y)_{y \leqq f(x)} R(T, x, y)\right]
$$

has measure 1. Unfortunately, D. A. Martin has shown that for some recursive $R$ this stronger proposition is false. We have to go to the language of arithmetic to realize the full flavor of measure-theoretic uniformity. Theorem 2.2 of $\$ 2$ states: let $B(T, x, y)$ be arithmetic [12]; then the set of all $T$ such that

$$
(x)(E y) B(T, x, y) \rightarrow(E f)\left[f \text { is arithmetic } \&(x)(E y)_{y \leqq f(x)} B(T, x, y)\right]
$$

has measure 1. In short, if we restrict $T$ to a set of measure 1 , then we can restrict ourselves, in our study of arithmetic predicates of $T$, to arithmetic bounding functions. The reduction from bounding functions arithmetic in $T$ to arithmetic bounding functions is the essence of measure-theoretic uniformity in the arithmetic case.

2. The arithmetic hierarchy. Let $B(T)$ be an arithmetic predicate of $T$. The set $\hat{T} B(T)$ is a Borel subset of $2^{N}$ of finite rank and consequently is measurable. Let $r$ 
be a variable ranging over the rational numbers in the unit interval $[0,1]$. By making use of some standard Gödel numbering, it is possible to regard the predicate $\mu(\hat{T} B(T)) \leqq r$ as a number-theoretic predicate whose free variables are $B$ (ranging over the arithmetic predicates) and $r$ (ranging over the rationals in $[0,1]$ ). For each $n>0$, an arithmetic predicate is said to belong to $\Sigma_{n}^{0}\left(\Pi_{n}^{0}\right)$ if it is expressible by means of a recursive matrix and a quantifier prefix beginning with an existential (universal) quantifier and passing through $n-1$ alternations of quantifiers. Recursive predicates are said to belong to both $\Sigma_{0}^{0}$ and $\Pi_{0}^{0}$.

LEMMA 2.1. (i) For each $n \geqq 0$ : the predicate $\mu(\hat{T} B(T)) \leqq r$, restricted to $B \in \Sigma_{n}^{0}$, is $\Pi_{n}^{0}$.

(ii) For each $n \geqq 0$ : the predicate $\mu(\hat{T} B(T)) \leqq r$, restricted to $B \in \Pi_{n}^{0}$, is $\Pi_{n+1}^{0}$.

(iii) The predicate $\mu(\hat{T} B(T)) \leqq r$, where $B$ is arithmetic and otherwise unrestricted, is hyperarithmetic.

Proof. (i) and (ii) are proved simultaneously by induction on $n$. First let $n=0$. Then $B(T)$ is a recursive predicate, and there exists a finite union $\bigcup\left\{b_{i} \mid i<k\right\}$ of basic open subsets of $2^{N}$ such that

$$
B(T) \leftrightarrow(E i)_{i<k}\left(T \in b_{i}\right)
$$

for all $T$; furthermore, the finite union $\bigcup\left\{b_{i} \mid i<k\right\}$ can be found effectively from a Gödel number of $B(T)$. Clearly, $\mu(\hat{T} B(T))=\mu\left(\bigcup\left\{b_{i} \mid i<k\right\}\right)$. Now suppose $n>0$ and $B(T) \in \Sigma_{n}^{0}$. Then $B(T)$ is of the form $(E x) B(T, x)$, where $B(T, x) \in \Pi_{n-1}^{0}$, and

$$
\mu(\hat{T} B(T)) \leqq r \leftrightarrow(m)\left[\mu\left(\hat{T}\left((E x)_{x \leqq m} B(T, x)\right)\right) \leqq r\right] .
$$

The inductive hypothesis for (ii) implies that the right side of the above formula is $\Pi_{n}^{0}$. Suppose instead that $B(T) \in \Pi_{n}^{0}$. Then $B(T)$ is of the form $(x) B(T, x)$, where $B(T, x) \in \Sigma_{n-1}^{0}$, and

$$
\mu(\hat{T} B(T)) \leqq r \leftrightarrow(\delta)(E m)\left[\mu\left(\hat{T}\left((x)_{x \leqq m} B(T, x)\right)\right) \leqq r+\delta\right],
$$

where $\delta$ is a variable ranging over the positive rationals. The inductive hypothesis for (i) implies that the right side of this last formula is $\Pi_{n+1}^{0}$.

To see (iii) observe that the "effective" nature of the inductive step of the proof of (i) and (ii) implies the existence of a recursive function $f$ with the following property: for each $n \geqq 0, f(n)$ is the Gödel number of a $\Pi_{n}^{0}$ predicate $P_{f(n)}(b, r)$ such that

$$
P_{f(n)}(b, r) \leftrightarrow b \text { is the Gödel number of } B(T) \& B(T) \in \Sigma_{n}^{0} \& \mu(\hat{T} B(T)) \leqq r .
$$

The predicate $P_{f(n)}(b, r)$, with $n, b$ and $r$ as free variables, is hyperarithmetic, since the truth set for arithmetic is hyperarithmetic.

In Theorem 2.2 we attempt to capture what we mean by measure-theoretic uniformity in the arithmetic case. A standard uniformity for arithmetic predicates of the form $B(T, x, y)$ can be put as follows: if $(x)(E y) B(T, x, y)$, then $(E f)[f$ is 
arithmetic in $T \&(x)(E y)_{y \leqq f(x)} B(T, x, y)$ ]. The corresponding measure-theoretic uniformity is obtained by demanding that the bounding function $f$ be arithmetic rather than arithmetic in $T$. The cost of such a demand is a set of $T$ 's of measure 0 .

THEOREM 2.2. Let $B(T, x, y)$ be arithmetic. Then the set of all T's satisfying the following condition has measure 1: if $(x)(E y) B(T, x, y)$, then $(E f)[f$ is arithmetic \& $\left.(x)(E y)_{y \leqq f(x)} B(T, x, y)\right]$.

Proof. Let $\delta$ be a positive rational. We define an arithmetic $f$ such that

$$
\mu\left(\hat{T}(x)(E y)_{y \leqq f(x)} B(T, x, y)\right)+\delta \geqq \mu(\hat{T}(x)(E y) B(T, x, y)) .
$$

Let $b(x, r)$ be the predicate: $\mu(\hat{T}(E y) B(T, x, y))>r$. By Lemma $2.1, b(x, r)$ is arithmetic. Then $f(x)$ is the least $j$ such that for all $r$,

$$
b(x, r) \rightarrow \mu\left(\hat{T}(E y)_{y \leqq} B(T, x, y)\right)>r-\delta / 2^{x+1} .
$$

COROLlaRY 2.3. If $B(T)$ is arithmetic and the set $\hat{T} B(T)$ has positive measure, then $B(A)$ holds for some arithmetic $A\left({ }^{3}\right)$.

Proof. First we consider a special case, where $B(T)$ is $\Pi_{2}^{0}$, and then we show how to reduce the general case to the special case. Let $B(T)$ be $(x)(E y) R(T, x, y)$, where $R(T, x, y)$ is recursive. By Theorem 2.2, there is an arithmetic function $f$ such that

$$
\mu\left(\hat{T}(x)(E y)_{y \leqq f(x)} R(T, x, y)\right)>0 .
$$

There exists an arithmetic function $c_{x}$ such that for each $x, c_{x}$ is a finite union of basic open subsets of $2^{N}$ and

$$
(E y)_{y \leqq f(x)} R(T, x, y) \leftrightarrow T \in c_{x} .
$$

For each $x$, let $d_{x}=\bigcap\left\{c_{i} \mid i \leqq x\right\}$. Note that for each $x$, the closed set $d_{x}$ is nonempty, because $\mu(\hat{T} B(T))>0$. If $A$ is an arithmetic set such that $(x)\left(A \in d_{x}\right)$, then $B(A)$ holds.'We define such an $A$ by an induction on $n$ :

$$
n \in A \leftrightarrow \mu\left(\hat{T}\left((x)\left(T \in d_{x}\right) \&(i)_{i<n}(i \in T \leftrightarrow i \in A) \& n \in T\right)\right)>0 .
$$

$A$ is arithmetical, since it is arithmetic in the function $d_{x}$.

Now we show how to reduce the general case to the special case by considering an illustrative example. Let $B(T)$ be $(x)(E y)(u)(E v) R(T, x, y, u, v)$, where $R(T, x, y, u, v)$ is recursive. By Theorem 2.2, there is an arithmetic $f$ such that

$$
\hat{T}(x)(E y)_{y \leqq f(x)}(u)(E v) R(T, x, y, u, v)
$$

has positive measure. By using a trick of Kleene [12], we can pass a bounded existential quantifier through a universal quantifier:

$$
(E y)_{y \leqq f(x)}(u)(E v) R(T, x, y, u, v) \leftrightarrow(u)(E y)_{y \leqq f(x)}(E v) R\left(T, x, y,(u)_{y}, v\right) .
$$

(3) Corollary 2.3 was independently obtained by H. Tanaka [34]. 
By Theorem 2.2, there is an arithmetic $g$ such that the set

$$
\hat{T}(x)(u)(E v)_{v \leqq g(x, u)}(E y)_{y \leqq f(x)} R\left(T, x, y,(u)_{y}, v\right)
$$

has positive measure. But now the argument of the special case can be applied.

Let us say that $B(T)$ holds for almost all $T$ if $\mu(\hat{T} B(T))=1$. Then Corollary 2.3 is equivalent to the statement: if $B(T)$ is arithmetic and holds for all arithmetic $T$, then it holds for almost all $T$. It follows immediately that the predicate " $T$ is arithmetic" is not arithmetic. This last result was first proved by Addison [1] by means of a forcing argument.

Corollary 2.3 is an example of what might be called a measure-theoretic basis result. Gandy's basis theorem [7] for arithmetic predicates states: if $B(T)$ is arithmetic and $(E T) B(T)$, then $B(A)$ holds for some $A$ recursive in Kleene's $O$, the set of all notations for recursive ordinals. (Kleene [13] gives an example of an arithmetic predicate $B(T)$ such that $(E T) B(T)$ but $B(T)$ holds for no hyperarithmetic $T$.)

Call a set $T$ fundamental (with respect to arithmetic) if $B(T)$ holds for every arithmetic $B$ such that $\mu(\hat{T} B(T))=1$. It is possible to construct hyperarithmetic, fundamental (with respect to arithmetic) sets with the help of Lemma 2.1, but we prefer to deduce the existence of such sets as a corollary to Theorem 3.9. They behave very much like the generic (with respect to arithmetic) sets of Feferman [3]. In particular, they are not implicitly arithmetically definable; that is, they are not unique solutions of arithmetic predicates.

3. The hyperarithmetic hierarchy. An analytic predicate $P(T, x)$ (Kleene [12]) is said to be $\Pi_{1}^{1}$ if it is expressible in the form $(Y) A(Y, T, x)$, where $A(Y, T, x)$ is arithmetic. Let $P(T, x, y)$ be $\Pi_{1}^{1}$. A familiar uniformity due to Kreisel [16] can be put as follows: if $(x)(E y) P(T, x, y)$, then

$$
(E f)\left[f \text { is hyperarithmetic in } T \&(x)(E y)_{y \leqq s(x)} P(T, x, y)\right] \text {. }
$$

The corresponding measure-theoretic uniformity is obtained by first discarding a set of $T$ 's of measure 0 and then requiring that the bounding function $f$ be hyperarithmetic rather than hyperarithmetic in $T$. We develop this result (Corollary 3.12) and several related ones by studying a relativization of Kleene's ramified analytic hierarchy [14]. Very briefly, the ramified analytic hierarchy is obtained by starting with the empty set and iterating the process of analytic definability through the recursive ordinals $\left({ }^{4}\right)$. Kleene [14] showed that the ramified analytic hierachy is identical with the set of hyperarithmetic sets. Following Feferman [3], we relativize by starting with an arbitrary set $T$ rather than the empty $\operatorname{set}\left({ }^{5}\right)$.

( ${ }^{4}$ Many persons now use the term "ramified analytic hierarchy" to denote the result of iterating the process of analytic definability past the recursive ordinals to its endpoint $\beta_{0}$.

${ }^{5}$ ) It might seem more natural to relativize by replacing not only the empty set by $T$ but also the recursive ordinals by the ordinals recursive in $T$; however, it follows from Lemma 3.5 that this relativization is identical with the one we adopted for almost all $T$. 
The relativization is described by a ramified, second-order language $\mathscr{L}(\mathscr{T})$, which differs little from Feferman's language $\mathscr{L}^{*}(\mathscr{S})([3$, p. 335]). $\mathscr{L}(\mathscr{T})$ is the language of first-order number theory augmented by the constant symbol $\mathscr{T}$ denoting an arbitrary set of natural numbers, some second-order set variables, and the membership symbol $(\epsilon)$. Let $O_{1}$ be a $\Pi_{1}^{1}$ subset of $O$ such that $O_{1}$ is linearly ordered by $<_{0}$ and has order-type $\omega_{1}$ (the least nonrecursive ordinal). (The existence of $O_{1}$ is proved in [4] and in [6].) Thus each recursive ordinal has just one notation in $O_{1}$; if $b$ is the unique notation in $O_{1}$ for the recursive ordinal $\beta$, we write $|b|=\beta$. For each $b \in O_{1}, \mathscr{L}(\mathscr{T})$ has ranked set variables $X^{b}, Y^{b}, Z^{b}, \ldots$; $\mathscr{L}(\mathscr{T})$ also has unranked set variables $X, Y, Z, \ldots$, a numeral $\bar{n}$ for each natural number $n$, and symbols for equality $(=)$, successor $\left({ }^{\prime}\right)$, addition $(+)$, and multiplication $(\cdot)$.

A formula $\mathscr{F}$ of $\mathscr{L}(\mathscr{T})$ is said to be ranked if every set variable occurring in $\mathscr{F}$ is ranked. The ordinal rank of a ranked sentence $\mathscr{F}$ is the least $\alpha$ such that $\alpha>|b|$ for every variable $X^{b}$ occurring in $\mathscr{F}$. Let $T$ be an arbitrary set of natural numbers. Following Feferman [3], for each $b \in O_{1}$, we inductively define a structure $\mathscr{M}_{b}(T)$ and truth in the structure $\bigcup\left\{\mathscr{M}_{a}(T)|| a|<| b \mid\right\}$ :

(i) A sentence $\mathscr{F}$ of ordinal rank $\leqq|b|$ is true in $\bigcup\left\{\mathscr{M}_{a}(T)|| a|<| b \mid\right\}$ if it is true when $\mathscr{T}$ is interpreted as $T$, the number variables of $\mathscr{F}$ are restricted to $\omega$, and each set variable $X^{a}$ of $\mathscr{F}$ is restricted to $\mathscr{M}_{a}(T)$.

(ii) For each formula $\mathscr{G}(x)$ (with only $x$ free) of ordinal rank $\leqq|b|$, let $\hat{x} \mathscr{G}(x)$ denote the set $\left\{n \mid \mathscr{G}(\bar{n})\right.$ is true in $\left.\bigcup\left\{\mathscr{M}_{a}(T)|| a|<| b \mid\right\}\right\} \mathscr{M}_{b}(T)$ consists of all sets of natural numbers defined in this manner.

We define $\mathscr{M}(T)=\bigcup\left\{\mathscr{M}_{b}(T) \mid b \in O_{1}\right\}$. Let $\mathscr{M}=\mathscr{M}(\phi)=$ the set of all hyperarithmetic sets. A sentence $\mathscr{F}$ of $\mathscr{L}(\mathscr{T})$ is true (in symbols, $\mathscr{M}(T) \vDash \mathscr{F}$ ) if it is true when each unranked variable is restricted to $\mathscr{M}(T)$ and the remaining symbols of $\mathscr{F}$ are interpreted according to (i) and (ii). It is a routine matter to choose a Gödel numbering for the formulas of $\mathscr{L}(\mathscr{T})$ with the following properties: the predicate " $e$ is the Gödel number of a ranked formula of $\mathscr{L}(\mathscr{T})$ " is $\Pi_{1}^{1}$; for each $b \in O_{1}$, the predicate " $e$ is the Gödel number of a ranked formula of $\mathscr{L}(\mathscr{T})$ of ordinal rank $<|b|$ " is recursively enumerable (uniformly in $b$ ). The assignment of Gödel numbers makes it possible to classify various relations involving the formulas of $\mathscr{L}(\mathscr{T})$. We will occasionally blur the distinction between formulas and Gödel numbers of formulas.

The full ordinal rank of a ranked formula $\mathscr{F}$ is a function $f: O_{1} \rightarrow \omega$ such that for each $b \in O_{1}, f(b)$ is the number of occurrences of $\left(X^{b}\right),\left(E Y^{b}\right), \ldots$ in $\mathscr{F}$. If $f$ and $g$ are full ordinal ranks, then $f<_{r} g$ if

$$
(E c)\left[c \in O_{1} \& f(c)<g(c) \&(d)\left(c<_{o_{1}} d \rightarrow f(d)=g(d)\right)\right] .
$$

The relation $<_{r}$ is a well-ordering of the set of full ordinal ranks.

If $\mathscr{G}(x)$ is a ranked formula (with only $x$ free), then $\mathscr{F}(\hat{x} \mathscr{G}(x)$ ) denotes the result of replacing each occurrence of $t \in Y$ in $\mathscr{F}(Y)$ by $\mathscr{G}(t)$. ( $t$ is a number-theoretic term.) 
Proposition 3.1. Let $\mathscr{F}\left(Y^{b}\right)$ be a ranked formula in prenex normal form whose only free variable is $Y^{b}$. Let $\left\{\mathscr{G}_{i}(x) \mid i \leqq n\right\}$ be a finite sequence of formulas with only. $x$ free of ordinal rank $\leqq|b|$. Then the disjunction $\bigvee_{i \leqq n} \mathscr{F}\left(\hat{x} \mathscr{G}_{i}(x)\right)$ is equivalent to $a$ prenex normal sentence of full ordinal rank less than that of $\left(E Y^{b}\right) \mathscr{F}\left(Y^{b}\right)$.

Proof. By illustrative example. Let $\mathscr{F}\left(Y^{b}\right)$ be

$$
\left(E X^{c}\right)\left(Y^{d}\right)\left(E Z^{b}\right) R\left(X^{c}, Y^{d}, Z^{b}, Y^{b}\right)
$$

where $R$ contains no quantifiers, and let $n=2$. The following formulas are equivalent:

$$
\begin{gathered}
\bigvee_{i \leqq 1}\left(E X^{c}\right)\left(Y^{d}\right)\left(E Z^{b}\right) R\left(X^{c}, Y^{d}, Z^{b}, \hat{x} \mathscr{G}_{i}(x)\right) ; \\
\left(E X^{c}\right)\left[\bigvee_{i \leqq 1}\left(Y^{d}\right)\left(E Z^{b}\right) R\left(X^{c}, Y^{d}, Z^{b}, \hat{x} \mathscr{G}_{i}(x)\right)\right] ; \\
\left(E X^{c}\right)\left(Y_{0}^{d}\right)\left(Y_{1}^{d}\right)\left[\bigvee_{i \leqq 1}\left(E Z^{b}\right) R\left(X^{c}, Y_{i}^{d}, Z^{b}, \hat{x} \mathscr{G}_{i}(x)\right)\right] ; \\
\left(E X^{c}\right)\left(Y_{0}^{d}\right)\left(Y_{1}^{d}\right)\left(E Z^{b}\right)\left[\bigvee_{i \leqq 1} R\left(X^{c}, Y_{i}^{d}, Z^{b}, \hat{x} \mathscr{G}_{i}(x)\right)\right]
\end{gathered}
$$

The block of quantifiers $\left(Y_{0}^{d}\right)\left(Y_{1}^{d}\right)$ can be replaced by a single quantifier $\left(Y^{d}\right)$ by means of a standard trick: each occurrence of $t \in Y_{0}^{d}\left(t \in Y_{1}^{d}\right)$ is replaced by $2 t$ $\in Y^{d}\left(2 t+1 \in Y^{d}\right)$. The formula

$$
\bigvee_{i \leqq 1} R\left(X^{c}, Y_{i}^{d}, Z^{b}, \hat{x} \mathscr{G}_{i}(x)\right)
$$

is equivalent to a prenex normal formula $Q\left(X^{c}, Y^{d}, Z^{b}\right)$ such that all of its quantifiers have superscripts which are notations for ordinals $<|b|$. Clearly,

$$
\left(E Y^{b}\right)\left(E X^{c}\right)\left(Y^{d}\right)\left(E Z^{b}\right) R\left(X^{c}, Y^{d}, Z^{b}, Y^{b}\right)
$$

has greater full ordinal rank than

$$
\left(E X^{c}\right)\left(Y^{d}\right)\left(E Z^{b}\right) Q\left(X^{c}, Y^{d}, Z^{b}\right)
$$

For each sentence $\mathscr{F}$ of $\mathscr{L}(\mathscr{T})$, we define $p(\mathscr{F})$, the probability that $\mathscr{F}$ is true in $\mathscr{M}(T)$, to be the Lebesgue measure of $\{T \mid \mathscr{M}(T) \vDash \mathscr{F}\}$. (It is easily seen by transfinite induction that $\{T \mid \mathscr{M}(T) \vDash \mathscr{F}\}$ is a Borel subset of $2^{N}$.)

LEMMA 3.2. The predicate $p(\mathscr{F}) \geqq r$, restricted to ranked $\mathscr{F}$ and rational $r$, is $\Pi_{1}^{1}$.

Proof. Spector observed that the intersection of all sets satisfying a $\Sigma_{1}^{1}$ condition is $\Pi_{1}^{1}$; i.e., if $A(X)$ is $\Sigma_{1}^{1}$, then $\bigcap\{X \mid A(X)\}$ is $\Pi_{1}^{1}$. We give an inductive definition of $p(\mathscr{F}) \geqq r$, restricted to ranked, prenex normal $\mathscr{F}$ and rational $r$, and then we note that the cases of our inductive definition can be expressed by means of $\Sigma_{1}^{1}$ closure conditions. The number-quantifier rank of $\mathscr{F}$ is the number of occurrences of $(x),(E y), \ldots$ in $\mathscr{F}$. The rank of $\mathscr{F}$ is the ordered pair $(f, m)$, where $f$ is the full ordinal rank of $\mathscr{F}$ and $m$ is the number-quantifier rank of $\mathscr{F}$. We say $(f, m)<(g, n)$ 
if $f<_{r} g$ or if $f=g$ and $m<n$. The inductive definition of $p(\mathscr{F}) \geqq r$ proceeds according to the rank of $\mathscr{F}$.

Case $0 . \mathscr{F}$ has no quantifiers. The predicate $p(\mathscr{F}) \geqq r$, restricted to $\mathscr{F}$ without quantifiers and rational $r$, is recursive; i.e., $p(\bar{m} \in \mathscr{T})=\frac{1}{2}$ etc.

Case 1. $\mathscr{F}$ is of the form $\left(E X^{b}\right) \mathscr{F}_{1}\left(X^{b}\right)$. Let $\left\{\mathscr{G}_{i}(x) \mid i \geqq 0\right\}$ be the uniform (in $b$ ) recursive enumeration of formulas with only $x$ free of ordinal rank $\leqq|b|$. Then $p(\mathscr{F}) \geqq r$ is defined to be

$$
(\delta)(E m)\left[p\left(\bigvee_{i \leqq m} \mathscr{F}_{1}\left(\hat{x} \mathscr{G}_{i}(x)\right)\right) \geqq r-\delta\right],
$$

where $\delta$ is restricted to the positive rationals. By $3.1, \bigvee_{i \leq m} \mathscr{F}_{1}\left(\hat{x} \mathscr{G}_{i}(x)\right)$ is equivalent to a prenex normal sentence of full ordinal rank less than that of $\left(E X^{b}\right) \mathscr{F}_{1}\left(X^{b}\right)$.

Case 2. $\mathscr{F}$ is of the form $\left(X^{b}\right) \mathscr{F}_{1}\left(X^{b}\right)$. Then $p(\mathscr{F}) \geqq r$ is defined to be

$$
(m)\left[p\left(\bigwedge_{i \leqq m} \mathscr{F}_{1}\left(\hat{x} \mathscr{G}_{i}(x)\right)\right) \geqq r\right] .
$$

By the dual of $3.1, \bigwedge_{i \leqq m} \mathscr{F}_{1}\left(\hat{x}_{\mathscr{G}_{i}}(x)\right)$ is equivalent to a prenex normal sentence of full ordinal rank less than that of $\left(X^{b}\right) \mathscr{F}_{1}\left(X^{b}\right)$.

Case 3. $\mathscr{F}$ is of the form $(E x) \mathscr{F}_{1}(x)$. Then $p(\mathscr{F}) \geqq r$ is defined to be

$$
(\delta)(E m)\left[p\left(\bigvee_{i \leqq m} \mathscr{F}_{1}(i)\right) \geqq r-\delta\right],
$$

where $\delta$ is restricted to the positive rationals. By the same argument used to establish 3.1, $\bigvee_{i \leqq m} \mathscr{F}_{1}(\bar{l})$ is equivalent to a prenex normal sentence of lower rank than that of $(E x) \mathscr{F}_{1}(x)$; in fact, the full ordinal rank will be unchanged but the number-quantifier rank will be less.

Case 4. $\mathscr{F}$ is of the form $(x) \mathscr{F}_{1}(x)$. Dual to Case 3.

Case 2 can be rephrased as a $\Sigma_{1}^{1}$ closure condition as follows: If $\mathscr{F}$ is a ranked, prenex normal sentence of the form $\left(X^{b}\right) \mathscr{F}_{1}\left(X^{b}\right)$ and $(m)\left[p\left(\bigvee_{i \leqq m} \mathscr{F}_{1}\left(\hat{x} \mathscr{G}_{i}(x)\right)\right) \geqq r\right]$, where $\left\{\mathscr{G}_{i}(x) \mid i \geqq 0\right\}$ is the uniform (in $b$ ) recursive enumeration of formulas with only $x$ free of rank $\leqq|b|$, then $p(\mathscr{F}) \geqq r$. The hypothesis of Case 2 is $\Pi_{1}^{1}$ in variables $\mathscr{F}$ and $r$.

LEMMA 3.3. Let $\mathscr{F}(x, Y)$ be a formula of $\mathscr{L}(\mathscr{T})$ whose only free variables are $x$ and $Y$, and whose only unranked variable is $Y$. Then for each rational $r$, there is a $c \in O_{1}$ such that

$$
p((x)(E Y) \mathscr{F}(x, Y)) \geqq r \rightarrow p\left((x)\left(E Y^{c}\right) \mathscr{F}\left(x, Y^{c}\right)\right) \geqq r\left(^{6}\right) .
$$

Proof. Fix $r$ and suppose $p((x)(E Y) \mathscr{F}(x, Y)) \geqq r$. Define $(Y)_{n}$ to be $\left\{i \mid p_{n}^{1+i} \in Y\right\}$, where $p_{n}$ is the $n$th smallest prime $\left(p_{0}=2, p_{1}=3, \ldots\right)$. Then

$$
(m)\left[p\left((E Y)\left(\bigwedge_{n \leqq m} \mathscr{F}\left(\bar{n},(Y)_{n}\right)\right)\right) \geqq r\right]
$$

${ }^{6}$ ) It is not possible in general to improve 3.3 by finding a $c$ that is independent of $r$. 
It follows that

$$
(m)(\delta)(E c)\left[c \in O_{1} \& p\left(\left(E Y^{c}\right)\left(\bigwedge_{n \leqq m} \mathscr{F}\left(\bar{n},\left(Y^{c}\right)_{n}\right)\right)\right) \geqq r-\delta\right],
$$

where $\delta$ is restricted to the positive rationals. It follows from 3.2 that the predicate

$$
\left[c \in O_{1} \& p\left(\left(E Y^{c}\right)\left(\bigwedge_{n \leqq m} \mathscr{F}\left(\bar{n},\left(Y^{c}\right)_{n}\right)\right)\right) \geqq r-\delta\right]
$$

is $\Pi_{1}^{1}$ in $c, m$ and $\delta$. By Kreisel's Lemma [16, p. 307], there exists a hyperarithmetic function $c(m, \delta)$ such that

$$
(m)(\delta)\left[p\left(\left(E Y^{c(m, \delta)}\right)\left(\bigwedge_{n \leqq m} \mathscr{F}\left(\bar{n},\left(Y^{c(m, \delta)}\right)_{n}\right)\right)\right) \geqq r-\delta\right] .
$$

By Spector [31] there is a $c \in O_{1}$ such that $(m)(\delta)(|c(m, \delta)| \leqq|c|)$. But then

$$
(m)(\delta)\left[p\left(\left(E Y^{c}\right)\left(\bigwedge_{n \leqq m} \mathscr{F}\left(\bar{n},\left(Y^{c}\right)_{n}\right)\right) \geqq r-\delta\right],\right.
$$

and consequently, $p\left((x)\left(E Y^{c}\right) \mathscr{F}\left(x, Y^{c}\right)\right) \geqq r$.

By an instance of the hyperarithmetic comprehension axiom, Kreisel [16] means the universal closure of any formula of the form

$$
(x)[(E Y) A(x, Y) \leftrightarrow(Z) B(x, Z)] \rightarrow(E X)(x)[x \in X \leftrightarrow(E Y) A(x, Y)],
$$

where $A(x, Y)$ and $B(x, Z)$ are arithmetic predicates that may contain free set variables other than $Y$ and $Z$.

LEMMA 3.4. If $\mathscr{F}$ is an instance of the hyperarithmetic comprehension axiom, then $p(\mathscr{F})=1$.

Proof. Let $A(x, Y)$ and $B(x, Y)$ be formulas of $\mathscr{L}(\mathscr{T})$ whose only free variables are $x$ and $Y$ and whose only unranked variable is $Y$. Let $K$ be the set of all $T$ such that $(x)[(E Y) A(x, Y) \leftrightarrow(Z) B(x, Z)]$ is true in $\mathscr{M}(T)$. Then $(x)(E Y)[A(x, Y)$ $\vee \sim B(x, Y)]$ is true in $\mathscr{M}(T)$ for all $T \in K$. Fix $\delta>0$. By 3.3 there is a $c \in O_{1}$ such that $(x)\left(E Y^{c}\right)\left[A\left(x, Y^{c}\right) \vee \sim B\left(x, Y^{c}\right)\right]$ is true in $\mathscr{M}(T)$ for all $T \in J \subseteq K$, where $\mu(K-J)<\delta$. But then $(x)\left[\left(E Y^{c}\right) A\left(x, Y^{c}\right) \leftrightarrow(E Y) A(x, Y)\right]$ holds in $\mathscr{M}(T)$ for all $T \in J$. Since $\left(E Y^{c}\right) A\left(x, Y^{c}\right)$ is a ranked formula with only $x$ free, it follows that $(E X)(x)[x \in X \leftrightarrow(E Y) A(x, Y)]$ holds in $\mathscr{M}(T)$ for all $T \in J$.

Lemma 3.4 suggests: if a set of $T$ 's of measure 0 is avoided, then $\mathscr{M}(T)$ is very similar to $\mathscr{M}$. The similarity is pursued further in Lemma 3.5 , and is exploited to prove 3.7. The underlying cause of the similarity is expressed by Lemma 3.2, which says that the probability that a ranked sentence $\mathscr{F}$ is true in $\mathscr{M}(T)$ can be "computed" in $\mathscr{M}$. The use of the term "computed" is appropriate for two closely related reasons: the predicate $p(\mathscr{F}) \geqq r$, restricted to ranked $\mathscr{F}$ and rational $r$, is $\Sigma_{1}^{1}$ in the sense of $\mathscr{M}$ (i.e., the existential set quantifier is restricted to the sets of $\mathscr{M})$; and the predicate is metarecursive [17] as well. 
LEMMA 3.5. For almost all $T, \mathscr{M}(T)$ is the set of all sets hyperarithmetic in $T\left({ }^{7}\right)$.

Proof. The argument of Kleene [14, p. 35], relativized to $T$, shows that the sets of $\mathscr{M}(T)$ are just those sets each of which is recursive in $H_{b}^{T}$ for some $b \in O_{1} \subseteq O^{T}\left({ }^{8}\right)$. According to Kreisel [15], if $T$ belongs to an ' $\omega$-model of the hyperarithmetic comprehension axiom, then every set hyperarithmetic in $T$ also belongs to that $\omega$-model. Now apply 3.4.

Proposition 3.6. Let $\left\{\mathscr{F}_{1}\right\}$ be a hyperarithmetic sequence of ranked sentences of $\mathscr{L}(\mathscr{T})$. Then there is a ranked sentence $\mathscr{F}$ such that for all $T, \mathscr{M}(T) \vDash \mathscr{F}$ if and only if $(i)\left[\mathscr{M}(T) \vDash \mathscr{F}_{i}\right]$.

Proof. Let $H$ be a hyperarithmetic set such that $n \in H \leftrightarrow n$ is the Gödel number of a member of $\left\{\mathscr{F}_{i}\right\}$. By Spector [31] there is a $c \in O_{1}$ such that the ordinal rank of $\mathscr{F}_{i}$ is $\leqq|c|$ for all $i$. Let $V_{c}^{T}$ be the set of Gödel numbers of all formulas of ordinal rank $\leqq|c|$ which are true in $\mathscr{M}(T)$. By adapting the argument of Kleene [14, p. 36], one can show that $V_{c}^{T}$ is recursive in $H_{c^{\prime}}^{T}$ for some $c^{\prime}$ in $O_{1} \subseteq O^{T}$ (see footnote ${ }^{8}$ )). Clearly, $(i)\left(i \in H \rightarrow i \in V_{c}^{T}\right)$ is equivalent to $(i)\left[\mathscr{M}(T) \vDash \mathscr{F}_{i}\right]$. Again by adapting the arguments of Kleene [14, p. 35], one can show the existence of ranked formulas $\mathscr{G}_{1}(x), \mathscr{G}_{2}(x)$, and $\mathscr{G}_{2}(x)$ of $\mathscr{L}(\mathscr{T})$ such that for all $T$ :

$$
\begin{aligned}
H & =\left\{n \mid \mathscr{M}(T) \vDash \mathscr{G}_{1}(\bar{n})\right\} ; \\
H_{c^{\prime}}^{T} & =\left\{n \mid \mathscr{M}(T) \vDash \mathscr{G}_{2}(\bar{n})\right\} ; \\
V_{c}^{T} & =\left\{n \mid \mathscr{M}(T) \vDash \mathscr{G}_{3}(\bar{n})\right\} .
\end{aligned}
$$

The desired $\mathscr{F}$ is $(x)\left(\mathscr{G}_{1}(x) \rightarrow \mathscr{G}_{3}(x)\right)$.

The Gödel number of $\mathscr{F}$ can be found effectively from the Gödel number of $\left\{\mathscr{F}_{i}\right\}$, since one can pass effectively from the Gödel number of $\left\{\mathscr{F}_{i}\right\}$ to $c, c^{\prime}$, and the Gödel numbers of $\mathscr{G}_{1}$ and $\mathscr{G}_{3}$.

LEMMA 3.7. For each ranked sentence $\mathscr{F}$ and rational $\delta>0$, there exists a ranked sentence $\mathscr{G}$ such that $p(\mathscr{F} \& \sim \mathscr{G})<\delta$ and such that $\{T \mid \mathscr{M}(T) \vDash \mathscr{G}\}$ is a closed subset of $\{T \mid \mathscr{M}(T) \vDash \mathscr{F}\}$.

Proof. Let $P(\mathscr{F}, \delta, \mathscr{G})$ denote the following number-theoretic predicate: $\mathscr{F}$ is a ranked sentence, $\delta$ is a positive rational, $\mathscr{G}$ is a ranked sentence, $p(\mathscr{F} \& \sim \mathscr{G})<\delta$, and $\{T \mid \mathscr{M}(T) \vDash \mathscr{G}\}$ is a closed subset of $\{T \mid \mathscr{M}(T) \vDash \mathscr{F}\}$. With the help of 3.2 and the fact that $\mathscr{M}(T) \vDash \mathscr{F}$, restricted to ranked $\mathscr{F}$, is $\Pi_{1}^{1}$, it is not hard to check that $P(\mathscr{F}, \delta, \mathscr{G})$ is $\Pi_{1}^{1}$.

We prove by induction on the rank of $\mathscr{F}$ that $(\delta)(E \mathscr{G}) P(\mathscr{F}, \delta, \mathscr{G})$. The cases are the same as those of 3.2. (We again assume that $\mathscr{F}$ is in prenex normal form.) The most

(7) Feferman [3] showed by means of a forcing argument with finite conditions that for all $T$ outside a first category subset of $2^{N}, \mathscr{H}(T)$ is the set of all sets hyperarithmetic in $T$.

$\left.{ }^{8}\right)$ There is no harm in thinking of $O_{1}$ as a subset of $O^{T}$, since there exists a recursive function $f$ with the following properties: for all $T, a<o_{o_{1}} b \leftrightarrow f(a)<_{o^{T}} f(b)$, and $a \in O_{1} \rightarrow|a|=$ $|f(a)|_{0^{T}}$. 
interesting case is when $\mathscr{F}$ is of the form $\left(X^{b}\right) \mathscr{F}_{1}\left(X^{b}\right)$. Let $\left\{\mathscr{G}_{i}(x) \mid i \geqq 0\right\}$ be the uniform (in $b$ ) recursive enumeration of formulas (with only $x$ free) of ordinal rank $\leqq|b|$. For each $i, \mathscr{H}_{1}\left(\hat{x} \mathscr{G}_{i}(x)\right)$ has lower full ordinal rank than $\left(X^{b}\right) \mathscr{F}_{1}\left(X^{b}\right)$. So by the induction hypothesis,

$$
(i)(\delta)(E \mathscr{H}) P\left(\mathscr{F}_{1}\left(\hat{x} \mathscr{G}_{i}(x)\right), \delta / 2^{i+1}, \mathscr{H}\right) .
$$

Since $P$ is $\Pi_{1}^{1}$, it follows from Kreisel's Lemma [16, p. 307] that there exists a hyperarithmetic sequence $\left\{\mathscr{H}_{i} \mid i \geqq 0\right\}$ of ranked formulas such that

$$
\text { (i) } P\left(\mathscr{F}_{1}\left(\hat{x} \mathscr{G}_{i}(x)\right), \delta / 2^{i+1}, \mathscr{H}_{i}\right) \text {. }
$$

By 3.6, there is a ranked formula $\mathscr{H}$ equivalent to the "infinite conjunction" of the $\mathscr{H}_{i}$ 's. Then $P(\mathscr{F}, \delta, \mathscr{H})$.

LEMMA 3.8. If $\mathscr{F}$ is a ranked sentence of $\mathscr{L}(\mathscr{T})$ and $p(\mathscr{F})>0$, then $\mathscr{M}(T) \vDash \mathscr{F}$ for some hyperarithmetic $T$.

Proof. By 3.7 it is safe to assume that $\{T \mid \mathscr{M}(T) \vDash \mathscr{F}\}$ is a closed subset of $2^{N}$. We inductively define a sequence $\left\{\mathscr{G}_{n} \mid n \geqq 0\right\}$ of atomic sentences:

$$
\begin{aligned}
\mathscr{G}_{n} & =\bar{n} \in \mathscr{T} & & \text { if } p\left(\mathscr{F} \&(i)_{i<n} \mathscr{G}_{i} \& \bar{n} \in \mathscr{T}\right)>0 \\
& =\bar{n} \notin \mathscr{T} & & \text { otherwise. }
\end{aligned}
$$

By 3.2 the set $H=\left\{n \mid \mathscr{G}_{n} \vDash \bar{n} \in \mathscr{T}\right\}$ is hyperarithmetic. Clearly, $\mathscr{M}(H) \vDash \mathscr{F}$.

THEOREM 3.9. If $P(X)$ is $\Pi_{1}^{1}$ and the Lebesgue measure of $\hat{X} P(X)$ is positive, then $P(H)$ holds for some hyperarithmetic $H\left({ }^{9}\right)$.

Proof. By Gandy [6] or Spector [33], there is an arithmetic predicate $\mathscr{A}(X, Y)$ such that

$$
(X)[P(X) \leftrightarrow(E Y)(Y \text { is hyperarithmetic in } X \& \mathscr{A}(X, Y)] \text {. }
$$

It follows from 3.5 that for almost all $T$,

$$
[\mathscr{M}(T) \vDash(E Y) \mathscr{A}(\mathscr{T}, Y)] \leftrightarrow P(T) ;
$$

consequently,

$$
p((E Y) \mathscr{A}(\mathscr{T}, Y))=\mu(\hat{X} P(X))>0 .
$$

By 3.8, $\mathscr{M}\left(H_{1}\right) \vDash(E Y) \mathscr{A}(\mathscr{T}, Y)$ for some hyperarithmetic $H_{1}$. Since every member of $\mathscr{M}\left(H_{1}\right)$ is hyperarithmetic in $H_{1}$, there must be a hyperarithmetic $H \in \mathscr{M}\left(H_{1}\right)$ such that $\mathscr{A}\left(H_{1}, H\right)$, and hence $P(H)$, hold.

Theorem 3.9 is another example of what might be called a measure-theoretic basis theorem. Shoenfield's basis result [27] for $\Pi_{1}^{1}$ predicates states: if $P(X)$ is $\Pi_{1}^{1}$ and $(E X) P(X)$, then $P(C)$ holds for some $C$ constructible in the sense of Gödel [9].

(9) Theorem 3.9 first appeared in Sacks [24]. Subsequently, H. Tanaka [34] independently developed another proof of 3.9 in which Corollary 5 of Sacks [23] (Corollary 3.10 of the present paper) is quoted but which avoids the formalism of the ramified analytic hierarchy. 
Theorem 3.9 can be relativized in a reasonable manner. Let $P(A, X)$ be $\Pi_{1}^{1}$ with $A$ and $X$ as free set variables. For each $A$, if the Lebesgue measure of $\hat{X} P(A, X)$ is positive, then $P(A, H)$ holds for some $H$ hyperarithmetic in $A$. It follows that if a complementary analytic set of reals [28] has positive Lebesgue measure, then it contains a real number hyperarithmetic in the determining system [28, p. 34] of its complement.

Another way of expressing Theorem 3.9 is: if $P(X)$ is $\Pi_{1}^{1}$ and holds for every hyperarithmetic $X$, then $P(X)$ holds for almost every $X$. We have suppressed a considerable amount of uniformity inherent in 3.9. It is not difficult to check: there is a partial recursive function $f$ such that if $e$ is the Gödel number of a $\Pi_{1}^{1}$ predicate $P(X)$ and the Lebesgue measure of $\hat{X} P(X)$ is positive, then $f(e)$ is defined and is the Gödel number of a hyperarithmetic set $H$ such that $P(H)$ holds.

The least ordinal not recursive in $X$ is denoted by $\omega_{1}^{X}$, the least nonrecursive ordinal by $\omega_{1}$.

\section{COROLlary 3.10. The set $\left\{X \mid \omega_{1}^{X}=\omega_{1}\right\}$ has Lebesgue measure 1.}

Proof. First observe that $\omega_{1}^{X}>\omega_{1}$ is $\Pi_{1}^{1}$. By Gandy [6] or Feferman-Spector [4] there is a recursive linear ordering $<_{R}$ of the natural numbers whose maximal well-ordered initial segment has order-type $\omega_{1}$. Then $\omega_{1}^{X}>\omega_{1}$ is equivalent to the following $\Pi_{1}^{1}$ predicate: $(E e)[e$ is the Gödel number of a recursive-in- $X$ well-ordering of the natural numbers $\left.\left(<_{e}\right)\right] \&(f)\left[f\right.$ is not a 1-1 order-preserving map of $<_{e}$ onto an initial segment of $<_{R}$ ]. Now by Spector [31], $\omega_{1}^{H}=\omega_{1}$ for all hyperarithmetic $H$. Then by $3.9, \omega_{1}^{X}=\omega_{1}$ for almost all $X$.

Corollary 3.11. If $A$ is not hyperarithmetic, then the set $\{X \mid A$ is hyperarithmetic in $X\}$ has Lebesgue measure $0\left({ }^{10}\right)$.

Proof. Suppose $\mu\left(\left\{X \mid A \leqq_{h} X\right\}\right)>0$. By 3.5, $\mu(\{T \mid A \in \mathscr{M}(T)\})>0$. There must then exist a ranked formula $\mathscr{G}(x)$ such that

$$
\mu(\{T \mid A=\{n \mid \mathscr{M}(T) \vDash \mathscr{G}(\bar{n})\}\})>0 .
$$

Let $K \subseteq\{T \mid A=\{n \mid \mathscr{M}(T) \vDash \mathscr{G}(\bar{n})\}\}$ be such that $\mu(K)=r>0$, where $r$ is rational. Let $J$ be a finite union of basic open subsets of $2^{N}$ such that $\mu(K \Delta J)<r / 3$. (KAJ $=(K-J) \cup(J-K)$.) Let $\mathscr{J}$ be a ranked sentence of $\mathscr{L}(\mathscr{T})$ with the property that

$$
(T)[T \in J \leftrightarrow \mathscr{M}(T) \vDash \mathscr{J}]
$$

Then

$$
n \in A \leftrightarrow p(\mathscr{G}(\bar{n}) \& \mathscr{J}) \geqq 2 r / 3,
$$

and consequently, $A$ is hyperarithmetic by 3.2 .

$\left({ }^{10}\right)$ S. Kripke has greatly strengthened Corollary 3.11 in the following direction: let $K \subseteq 2^{N}$ be a set of measure 0 such that $\phi \notin K$ and such that any set of the same hyperdegree as some member of $K$ is also a member of $K$; then the set of all $X$ such that some $Y \in K$ is hyperarithmetic in $X$ has measure 0 . 
Corollary 3.11' (CARL JockusCh). Let $P(X)$ be $\Pi_{1}^{1}$. If $(Y)(Y \in H Y P \rightarrow \sim P(Y))$ then the Lebesgue measure of $\left\{X \mid(E Y)\left(P(Y) \& Y \leqq_{h} X\right)\right\}$ is 0 .

Proof. By 3.9 it is enough to show $(E Y)\left[P(Y) \& Y \leqq_{n} X\right]$ is $\Pi_{1}^{1}$. This last predicate is equivalent to $(E b)\left[b \in O^{X} \& Y\right.$ is recursive in $\left.H_{b}^{X} \& P(Y)\right]$.

A set $A$ is said to be implicitly arithmetically definable if it is the unique solution of some arithmetic predicate; i.e., there is an arithmetic $B(X)$ such that $\left(E_{1} X\right) B(X)$ and $B(A)$. It is well known that each hyperarithmetic set is recursive in some implicitly arithmetically definable, hyperarithmetic set. Feferman [3] showed by means of a forcing argument with finite conditions that there exists a hyperarithmetic set which is not implicitly arithmetically definable. The notion of fundamental (with respect to arithmetic) was defined at the end of $\S 2$.

COROLlARY 3.12. There exists a hyperarithmetic set which is fundamental (with respect to arithmetic). Each such set is not implicitly arithmetically definable.

Proof. A set $T$ is fundamental (with respect to arithmetic) if for every arithmetic predicate $B(X)$,

$$
\mu(X B(X))=1 \rightarrow B(T) .
$$

By 2.2 (iii) and 3.9, there exists a hyperarithmetic $T$ which is fundamental with respect to arithmetic. If $B(X)$ is arithmetic and $B(T)$ holds, then $X B(X)$ must have positive Lebesgue measure.

THEOREM 3.13. Let $P(T, x, y)$ be $\Pi_{1}^{1}$. Then the set of all $T$ satisfying the condition below has Lebesgue measure 1:

$$
(x)(E y) P(T, x, y) \rightarrow(E f)_{H Y P}(x)(E y)_{y \leqq f(x)} P(T, x, y) .
$$

Proof. By Gandy [6] or Spector [33], there is an arithmetic formula $\mathscr{A}(T, x, y, Y)$ such that for all $T, m$, and $n$ :

$$
P(T, m, n) \leftrightarrow(E Y)\left[Y{ }_{n} T \& \mathscr{A}(T, m, n, Y)\right] .
$$

By 3.5, we have for almost all $T$ and all $m, n$ :

$$
P(T, m, n) \leftrightarrow \mathscr{M}(T) \vDash(E Y) \mathscr{A}(\mathscr{T}, \bar{m}, \bar{n}, Y) .
$$

Then for almost all $T$ :

$$
(x)(E y) P(T, x, y) \leftrightarrow \mathscr{M}(T) \vDash(x)(E y)(E Y) \mathscr{A}(\mathscr{T}, x, y, Y) .
$$

Let $\delta$ be a positive rational. We define a hyperarithmetic function $f$ such that the set of all $T$ satisfying the following condition has measure at least $1-\delta$ :

$$
(x)(E y) P(T, x, y) \rightarrow(x)(E y)_{y \leqq f(x)} P(T, x, y) .
$$

Let $b(n, r)$ be the predicate

$$
p((E y)(E Y) \mathscr{A}(\mathscr{T}, \bar{n}, y, Y))>r,
$$


where $r$ is rational. By $3.2, b(n, r)$ is hyperarithmetic. Then $f(n)$ is the least $j$ such that for all $r$,

$$
b(n, r) \rightarrow p\left((E y)_{y \leqq j}(E Y) \mathscr{A}(\mathscr{T}, n, y, Y)\right)>r-\delta / 2^{n+1} .
$$

Theorem 3.13 is the instance of measure-theoretic uniformity we associate with hyperarithmetic theory; by suppressing a set of $T$ 's of measure 0 , we are able to restrict our attention from bounding functions hyperarithmetic in $T$ (provided by Kreisel's Lemma [16]) to bounding functions that are actually hyperarithmetic. A sharpening of Theorem 3.13 will be useful.

Corollary 3.14. Let $P(T, x, y)$ and $Q(x, y)$ be $\Pi_{1}^{1}$ formulas such that $(x)(z)$ $\cdot(E y)_{z \leqq y} Q(x, y)$. Then the set of all $T$ satisfying the following condition has Lebesgue measure 1:

$(x)(E y)[P(T, x, y) \& Q(x, y)] \rightarrow(E f)_{H Y P}(x)(E y)_{y \leqq f(x)}[P(T, x, y) \& Q(x, f(x))]$.

Proof. By 3.13, for almost all $T$ there exists a hyperarithmetic $g$ such that

$$
(x)(E y)[P(T, x, y) \& Q(x, y)] \rightarrow(x)(E y)_{y \leqq g(x)}[P(T, x, y) \& Q(x, y)] .
$$

Since $(x)(z)(E y)_{z \leqq y} Q(x, y)$, it must be that

$$
(x)(E y)[g(x) \leqq y \& Q(x, y)] .
$$

By Kreisel's Lemma [16], there exists a hyperarithmetic $f$ such that

$$
(x)[g(x) \leqq f(x) \& Q(x, f(x))] .
$$

But then for almost all $T$ there exists a hyperarithmetic $f$ such that

$$
(x)(E y)[P(T, x, y) \& Q(x, y)] \rightarrow(x)(E y)_{y \leqq f(x)}[P(T, x, y) \& Q(x, f(x))] .
$$

Let $T_{0}, T_{1}, T_{2}, \ldots$ be an arbitrary sequence of sets of natural numbers. We turn our attention to the structure $\mathscr{M}\left(T_{0}, T_{1}, T_{2}, \ldots\right)$ introduced by Feferman [3]. The language $\mathscr{L}\left(\mathscr{T}_{0}, \mathscr{T}_{1}, \mathscr{T}_{2}, \ldots\right)$ differs from $\mathscr{L}(\mathscr{T})$ (described at the beginning of $\S 3$ ) only in that the set constant $\mathscr{T}$ has been replaced by the set constants $\mathscr{T}_{0}, \mathscr{T}_{1}, \mathscr{T}_{2}, \ldots ;$ the structure $\mathscr{M}\left(T_{0}, T_{1}, T_{2}, \ldots\right)$ is defined in the same manner as $\mathscr{M}(T)$. Each set constant $\mathscr{T}_{i}$ is of course interpreted as $T_{i}$. Each member of $\mathscr{M}_{b}\left(T_{0}\right.$, $\left.T_{1}, T_{2}, \ldots\right)$ is defined by a formula $\mathscr{G}(x)$ of ordinal rank $\leqq|b|$, where $b \in O_{1}$. Note that each such formula $\mathscr{G}(x)$ can explicitly mention only finitely many $\mathscr{T}_{i}$ 's; thus it is possible that $\mathscr{M}\left(T_{0}, T_{1}, T_{2}, \ldots\right)$ will not, in general, have a member in which all the $T_{i}$ 's are recursive.

We think of $\left(T_{0}, T_{1}, T_{2}, \ldots\right)$ as being a member of $\left(2^{N}\right)^{N}$. Lebesgue measure $\mu$ for $2^{N}$ was defined in $\S 1$ as the product measure induced by the unbiased measure $m$ on each factor $2=\{0,1\}$. Lebesgue measure $\mu$ for $\left(2^{N}\right)^{N}$ is defined similarly. If $\mathscr{F}$ is a sentence of $\mathscr{L}\left(\mathscr{T}_{0}, \mathscr{T}_{1}, \mathscr{T}_{2}, \ldots\right)$, then the set

$$
\left\{\left(T_{0}, T_{1}, \ldots\right) \mid \mathscr{M}\left(T_{0}, T_{1}, \ldots\right) \vDash \mathscr{F}\right\}
$$


is a Borel subset of $\left(2^{N}\right)^{N}$ and so is Lebesgue-measurable; let $p(\mathscr{F})$, the probability that $\mathscr{F}$ is true in $\mathscr{M}\left(T_{0}, T_{1}, \ldots\right)$, be the measure of this set.

Let $v$ and $w$ be recursive functions such that the map $h: N \rightarrow N \times N$, defined by $h(n)=(v(n), w(n))$, is one-to-one and onto. Let $x \in(T)_{y}$ be defined by

$$
(E n)[n \in T \& v(n)=x \& w(n)=y] \text {. }
$$

Then the map $h: 2^{N} \rightarrow\left(2^{N}\right)^{N}$ defined by $h(T)=\left((T)_{0},(T)_{1},(T)_{2}, \ldots\right)$ is one-to-one, onto, and measure-preserving; i.e., if $A \subseteq 2^{N}$, then $A$ is a Borel subset of $2^{N}$ of measure $m$ if and only if $h[A]$ is a Borel subset of $\left(2^{N}\right)^{N}$ of measure $m$ (see footnote $\left.\left({ }^{12}\right)\right)$.

LEMMA 3.15. The predicate $p(\mathscr{F}) \geqq r$, where $\mathscr{F}$ is restricted to the ranked sentences of $\mathscr{L}\left(\mathscr{T}_{0}, \mathscr{T}_{1}, \mathscr{T}_{2}, \ldots\right)$ and $r$ is rational, is $\Pi_{1}^{1}$.

Proof. Same as 3.2 .

By an instance of the $\Sigma_{1}^{1}$ axiom of choice, Kreisel [16] means the universal closure of any formula of the form:

$$
(x)(E Y) A(x, Y) \rightarrow(E Y)(x) A\left(x,(Y)_{x}\right),
$$

where $A(x, Y)$ is arithmetic and may contain free variables other than $x$ and $Y$. Feferman [3] showed that the $\Sigma_{1}^{1}$ axiom of choice holds in $\mathscr{M}\left(T_{0}, T_{1}, \ldots\right)$ for all $\left(T_{0}, T_{1}, \ldots\right)$ outside a first category subset of $\left(2^{N}\right)^{N}$ by means of a Cohen-type forcing argument.

THEOREM 3.16. If $\mathscr{F}$ is an instance of the $\Sigma_{1}^{1}$ axiom of choice, then $\mathscr{F}$ is true in $\mathscr{M}\left(T_{0}, T_{1}, T_{2}, \ldots\right)$ with probability 1 .

Proof. Let $\mathscr{A}(x, Y)$ be a formula of $\mathscr{L}\left(\mathscr{T}_{0}, \mathscr{T}_{1}, \ldots\right)$ whose only free variables are $x$ and $Y$, and whose only set variable is $Y$. Let $T$ be such that

$$
\mathscr{M}\left((T)_{0},(T)_{1}, \ldots\right) \vDash(x)(E Y) \mathscr{A}(x, Y) .
$$

We intend to show that almost every such $T$ has the property that

$$
\mathscr{M}\left((T)_{0},(T)_{1}, \ldots\right) \vDash(E Y)(x) \mathscr{A}\left(x,(Y)_{x}\right) .
$$

The lemma then follows from the measure-preserving property of the map $h(T)$ $=\left((T)_{0},(T)_{1}, \ldots\right)$. Let $\left\{\mathscr{G}_{b}(x) \mid b \in O_{1}\right\}$ be a $\Pi_{1}^{1}$ enumeration of all ranked formulas with only $x$ free of $\mathscr{L}\left(\mathscr{T}_{0}, \mathscr{T}_{1}, \mathscr{T}_{2}, \ldots\right)$. Then

$$
(n)(E b)\left[b \in O_{1} \& \mathscr{M}\left((T)_{0},(T)_{1}, \ldots\right) \vDash \mathscr{A}\left(\bar{n}, \hat{x} \mathscr{G}_{b}(x)\right)\right] .
$$

If $T$ lies outside a certain set of measure 0 , then it follows from 3.14 that there exists a hyperarithmetic function $f$ such that

$$
(n)(E b)_{b \leqq f(n)}\left[f(n) \in O_{1} \& \mathscr{M}\left((T)_{0},(T)_{1}, \ldots\right) \vDash \mathscr{A}\left(\bar{n}, \hat{x} \mathscr{G}_{b}(x)\right)\right]
$$

By Spector [31], there is a $c \in O_{1}$ such that $f(n)<{ }_{O_{1}} c$ for all $n$. Thus

$$
(n)(E b)\left[b \leqq o_{1} c \& \mathscr{M}\left((T)_{0},(T)_{1}, \ldots\right) \vDash \mathscr{A}\left(\bar{n}, \hat{x} \mathscr{G}_{b}(x)\right)\right]
$$


There exists a ranked formula $\mathscr{H}(x)$ of $\mathscr{L}\left(\mathscr{T}_{0}, \mathscr{T}_{1}, \mathscr{T}_{2}, \ldots\right)$ such that for all $T, n$, and $b \leqq o_{1} c$ :

$$
\mathscr{M}\left((T)_{0},(T)_{1}, \ldots\right) \vDash \mathscr{G}_{b}(\bar{n}) \leftrightarrow \mathscr{M}\left((T)_{0},(T)_{1}, \ldots\right) \vDash \bar{n} \in(\hat{x} \mathscr{H}(x))_{b} .
$$

The existence of $\mathscr{H}$ follows by the same argument used in 3.6. But then

$$
\mathscr{M}\left((T)_{0},(T)_{1}, \ldots\right) \vDash(x)(E y) \mathscr{A}\left(x,(\hat{x} \mathscr{H}(x))_{y}\right) .
$$

It easily follows that

$$
\mathscr{M}\left((T)_{0},(T)_{1}, \ldots\right) \vDash(E Y)(x) \mathscr{A}\left(x,(Y)_{x}\right) .
$$

The proof of Theorem 3.16 was little more than an application of Corollary 3.14, which was only a refinement of Theorem 3.13, the statement of measure-theoretic uniformity for the hyperarithmetic case. In the same manner, Lemma 3.4 and Theorem 3.9 can be viewed as consequences of Theorem 3.13. In short, if one keeps in mind the hyperarithmetic version of measure-theoretic uniformity, as expressed by Theorem 3.13 , one can readily recover all the results of $\S 3$.

4. The constructible hierarchy. Let $\mathscr{M}$ be a countable initial segment of $L$, the class of constructible sets, such that $\mathscr{M}$ is a model of ZF, the axioms of ZermeloFraenkel set theory, and hence of $V=L$, the axiom of constructibility. ( $\mathscr{M}=F^{\prime \prime} \alpha$ for some countable ordinal $\alpha$, where $F$ is defined in Gödel [9].) We utilize measuretheoretic ideas in the style of $\S 3$ to study the effect of adding an arbitrary set $T$ of natural numbers to $\mathscr{M}$. The resulting structure is denoted by $\mathscr{M}(T)$. Lemma 4.2 is the instance of measure-theoretic uniformity we associate with the constructible hierachy of $\mathscr{M}$, and it readily implies that the replacement axiom holds in $\mathscr{M}(T)$ with probability 1 . In order to give a complete proof of Lemma 4.1 , it is necessary to give some details concerning the structure of $\mathscr{M}(T)$. For this purpose the symbolism of Tharp [36] is convenient. The symbols of the language $\mathscr{L}^{0}$ are: $\in$ (membership); unranked set variables $x, y, z, \ldots$; ranked set variables $x^{\alpha}, y^{\alpha}, z^{\alpha}, \ldots$ for each ordinal $\alpha$ of $\mathscr{M}$; propositional connectives, and existential and universal quantifiers for both ranked and unranked set variables. The atoms of $\mathscr{L}^{0}$ are of the form $t_{1} \in t_{2}$, where $t_{1}$ and $t_{2}$ are variables. The formulas of $\mathscr{L}^{0}$ are constructed from the atoms and the logical symbols in the standard manner. We define a class $\mathscr{C}$ of constants (intended to name the members of $\mathscr{M}(T)$ ) by induction on the ordinals of $\mathscr{M}$.

$\mathscr{C}(0)=\{\bar{n} \mid n<\omega\}$.

$\mathscr{C}(\alpha+1): \mathscr{T}$ is a member of $\mathscr{C}(\alpha+1)$. Let $\phi\left(x^{\alpha}, y_{1}, \ldots, y_{n}\right)$ be a formula of $\mathscr{L}^{0}$ whose only free variables are $x^{\alpha}, y_{1}, \ldots, y_{n}(n \geqq 0)$, and whose only quantified variables are of the form $x^{\beta}$ for $\beta$ less than or equal to $\alpha$. Then $\hat{x}^{\alpha} \phi\left(x^{\alpha}, c_{1}, \ldots, c_{n}\right)$, where each $c_{i}(1 \leqq i \leqq n)$ is either $\mathscr{T}$ or a member of $\bigcup\{\mathscr{C}(\beta) \mid \beta \leqq \alpha\}$, is a typical member of $\mathscr{C}(\alpha+1)$.

$\mathscr{C}(\lambda)=\bigcup\{\mathscr{C}(\beta) \mid \beta<\lambda\}$ for each limit ordinal $\lambda$. 
Let $\mathscr{C}=\bigcup\{\mathscr{C}(\alpha) \mid \alpha \in \mathscr{M}\}$. The symbols of $\mathscr{L}(\mathscr{T})$ are those of $\mathscr{L}^{0}$ together with the members of $\mathscr{C}$. The atomic formulas of $\mathscr{L}(\mathscr{T})$ are of the form $t_{1} \in t_{2}$, where $t_{1}$ and $t_{2}$ are variables or members of $\mathscr{C}$.

A sentence $\mathscr{F}$ of $\mathscr{L}(\mathscr{T})$ is said to be ranked if all its variables are ranked. The ordinal rank of a ranked sentence $\mathscr{F}$, denoted by $o(\mathscr{F})$, is the least upper bound of $\left\{\beta \mid\left(x^{\beta}\right)\right.$ or $\left(E y^{\beta}\right)$ occurs in $\left.\mathscr{F}\right\} \cup\left\{\beta+1 \mid \hat{x}^{\beta}\right.$ occurs in $\left.\mathscr{F}\right\}$. If $t \in \mathscr{C}$, then the ordinal rank of $t$, denoted by $o(t)$, is the ordinal rank of the sentence $\overline{0} \in t$. For each set $T$ of natural numbers, we simultaneously define several concepts by induction on the ordinals of $\mathscr{M}: c \in \mathscr{C}(\alpha)$ and $b \in \mathscr{M}_{\alpha}(T)$ and $c$ denotes $b ; o(\mathscr{F}) \leqq \alpha$ and $\mathscr{F}$ is true in $\mathscr{U}_{\alpha}(T)$.

1. $\alpha=0 . \mathscr{M}_{0}(T)=\omega$ and $\bar{n}$ denotes $n$. $\mathscr{F}$ is true in $\mathscr{M}_{0}(T)$ if it is true when $\mathscr{T}$ is interpreted as $T, \bar{n}$ is interpreted as $n$, and the variables $x^{0}, y^{0}, z^{0}, \ldots$ are restricted to $\omega$.

2. $\alpha=\gamma+1$. Let $c \in \mathscr{C}(\alpha)$ be of the form $\hat{x}^{\gamma} \phi\left(x^{\gamma}\right)$. Then $c$ denotes $b$, where

$$
b=\left\{a \mid a \in \mathscr{M}_{\gamma}(T) \&(E d)\left[d \in \mathscr{C}(\gamma) \& d \text { denotes } a \& \mathscr{M}_{\gamma}(T) \vDash \phi(d)\right]\right\} .
$$

$\mathscr{M}_{\alpha}(T)$ is the set of all such $b$ 's. Let $o(\mathscr{F}) \leqq \alpha$. Then $\mathscr{F}$ is true in $\mathscr{M}_{\alpha}(T)$ if it is true when each quantified variable $x^{\beta}$ of $\mathscr{F}$ is restricted to $\mathscr{M}_{\beta}(T)$, and each constant $c$ of $\mathscr{C}(\beta)$ occurring in $\mathscr{F}$ is interpreted as the $b \in \mathscr{M}_{\beta}(T)$ it denotes.

3. $\alpha=\lambda$, where $\lambda$ is a limit ordinal. $\mathscr{M}_{\lambda}(T)=\bigcup\left\{\mathscr{M}_{\beta}(T) \mid \beta<\lambda\right\}$.

Let $\mathscr{M}(T)=\bigcup\left\{\mathscr{M}_{\alpha}(T) \mid \alpha \in \mathscr{M}\right\}$. Let $\mathscr{F}$ be an arbitrary sentence of $\mathscr{L}(\mathscr{T})$. $\mathscr{F}$ is true in $\mathscr{M}(T)(\mathscr{M}(T) \vDash \mathscr{F})$ if it is true when each unranked, quantified variable is restricted to $\mathscr{M}(T)$ and the remaining symbols are interpreted as above. If $\mathscr{F}$ is ranked, then $\mathscr{F}$ can be put in prenex normal form without any increase in ordinal rank. Assume $\mathscr{F}$ is in prenex normal form. The quantifier alternation rank of $\mathscr{F}$, denoted by $a(\mathscr{F})$, is the number of alternations of quantifiers in the prefix of $\mathscr{F}$. The leading quantifier rank of $\mathscr{F}$, denoted by $l(\mathscr{F})$, is the number of quantified variables occurring in the prefix of $\mathscr{F}$ on the left of the left-most alternation of quantifiers of $\mathscr{F}$ if $a(\mathscr{F})>0$. If $a(\mathscr{F})=0$, then $l(\mathscr{F})$ is the number of quantified variables occurring in the prefix of $\mathscr{F}$. Thus if $\mathscr{F}$ is $\left(x^{\alpha}\right)\left(x^{\beta}\right)\left(y^{\alpha}\right)\left(E y^{\nu}\right) \mathscr{G}$, where $\mathscr{G}$ has no quantifiers, then $l(\mathscr{F})=3$.

For each sentence $\mathscr{F}$, the countability of $\mathscr{M}$ implies that $\{T \mid \mathscr{M}(T) \vDash \mathscr{F}\}$ is a Borel subset of $2^{N}$. For each $\mathscr{F}$, the probability that $\mathscr{F}$ is true in $\mathscr{M}(T)$, denoted by $p(\mathscr{F})$, is defined to be the Lebesgue measure of the Borel set corresponding to $\mathscr{F}$. We have just given what might be called the external definition of the function $p(\mathscr{F})$. In Lemma 4.1 we give the internal definition, i.e., a definition inside $\mathscr{M}$. If $\mathscr{G}$ is an instance of the replacement axiom, then the fact that the replacement axiom is true in $\mathscr{M}$ together with the fact the probability function $p(\mathscr{F})$ is $\mathscr{M}$-definable will suffice to show $p(\mathscr{G})=1$. Hopefully, we will see this matter as a typical instance of the phenomenon of measure-theoretic uniformity. Of course the $\mathscr{M}$-definability of $p(\mathscr{F})$ plays the same role here as the $\mathscr{M}$-definability of the forcing relation in Cohen [2]. The difference, if there is one, is that $p(\mathscr{F})$ has a trivial external definition. 
Let $A$ be an arbitrary set. We say $A$ is $\mathscr{M}$-definable if there exists a formula $\mathscr{F}(x)$ of ZF with constants denoting elements of $\mathscr{M}$ such that $A$ equals

$$
\{a \mid a \in \mathscr{M} \& a \text { satisfies } \mathscr{F}(x) \text { in } \mathscr{M}\} \text {. }
$$

LEMMA 4.1. For each $n \geqq 0:$ The function $p(\mathscr{F})$, restricted to sentences $\mathscr{F}$ of $\mathscr{L}(\mathscr{T})$ having at most $n$ unranked quantifiers, is $\mathscr{M}$-definable.

Proof. We consider only $n=0$, since Cases 1 and 2 below make clear how to handle unranked quantifiers. We assume $\mathscr{F}$ is in prenex normal form and define $p(\mathscr{F})$ by induction on the rank of $\mathscr{F}=r(\mathscr{F})$, where $r(\mathscr{F})$ is the triple $(o(\mathscr{F}), a(\mathscr{F})$, $l(\mathscr{F})$ ). (The triples are ordered lexicographically.)

Case 1. $\mathscr{F}$ is $\left(x^{\alpha}\right) \mathscr{F}_{1}\left(x^{\alpha}\right)$. Then

$$
p(\mathscr{F})=\operatorname{glb}\left\{p\left(\bigwedge_{i \leqq n} \mathscr{F}_{1}\left(c_{i}\right)\right) \mid n<\omega, c_{i} \in \mathscr{C}(\alpha)\right\}
$$

where glb denotes "greatest lower bound". If $c_{i}(i \leqq n) \in \mathscr{C}(\alpha)$, then $\bigwedge_{i \leqq n} \mathscr{F}_{1}\left(c_{i}\right)$ can be put in prenex normal form $\mathscr{G}$ such that $o(\mathscr{G}) \leqq o(\mathscr{F})$. We claim $r(\mathscr{G})<r(\mathscr{F})$. If $l(\mathscr{F})>1$, then $a(\mathscr{G})=a(\mathscr{F})$ and $l(\mathscr{G})<l(\mathscr{F})$. If $l(\mathscr{F})=1$, then $a(\mathscr{G})<a(\mathscr{F})$. The last two assertions follow from the kind of elementary quantifier manipulation occurring in 3.1 .

Case 2. $\mathscr{F}$ is $\left(E x^{\alpha}\right) \mathscr{F}_{1}\left(x^{\alpha}\right)$. Dual to Case 1.

Case 3. $\mathscr{F}$ has no quantifier prefix. Thus $\mathscr{F}$ is a propositional combination of atoms of the form $t_{1} \in t_{2}$, where $t_{1}, t_{2} \in \mathscr{C}$. If every atom of $\mathscr{F}$ has ordinal rank 0 , then $p(\mathscr{F})$ is easily computed from the information given in Case 3a. If some atom of $\mathscr{F}$ has ordinal rank $>0$, then $\mathscr{F}$ is equivalent to a sentence of lower ordinal rank, as is indicated in Cases $3 \mathrm{~b}, 3 \mathrm{c}$ and $3 \mathrm{~d}$.

Case 3a. $p(\bar{m} \in \bar{n})=1$ if $m<n ; p(\bar{m} \in \mathscr{T})=\frac{1}{2} ; p(\mathscr{T} \in \bar{m})=p(\mathscr{T} \in \mathscr{T})=p(\bar{m} \in \bar{n})=0$ if $m \geqq n$.

Case 3b. $o\left(t_{1}\right)<o\left(t_{2}\right) . t_{2}$ must be of the form $\hat{x}^{\beta} \phi\left(x^{\beta}\right) . t_{1} \in t_{2}$ is equivalent to $\phi\left(t_{1}\right) . o\left(t_{1} \in t_{2}\right)=\beta+1$ and $\beta \geqq o\left(\phi\left(t_{1}\right)\right)$.

Case 3c. $o\left(t_{1}\right)>o\left(t_{2}\right)$. Then $t_{1} \in t_{2}$ is equivalent to

$$
\left(E y^{\alpha}\right)\left[\left(x^{\alpha}\right)\left(x^{\alpha} \in y^{\alpha} \leftrightarrow \phi\left(x^{\alpha}\right)\right) \& y^{\alpha} \in t_{2}\right],
$$

where $t_{1}$ is of the form $\hat{x}^{\alpha} \phi\left(x^{\alpha}\right) . o\left(t_{1} \in t_{2}\right)=\alpha+1$, but the ordinal rank of the above equivalent formula is $\leqq \alpha$.

Case 3d. $0<o\left(t_{1}\right)=o\left(t_{2}\right)$. Similar to Case $3 \mathrm{c}$.

Cases 1-3 constitute the definition of $p(\mathscr{F})$ "inside" $\mathscr{M}$. A routine induction shows the internal definition of the function $p(\mathscr{F})$ agrees with the external definition.

The proof of Lemma 4.1 relied heavily on the notion of prenex normal form. We comment further on this matter at the end of the paper. Lemma 4.2 is the instance of measure-theoretic uniformity we associate with the constructible hierarchy of $\mathscr{M}$. It is analogous to Theorems 2.2 and 3.13, but there is a difference worth noting. 
Both 2.2 and 3.13 have the form: for almost every $T$ there exists an $f$ etc., but 4.1 has the form: there exists an $f$ such that for almost all $T$ etc. The difference is a consequence of the strong "closure" properties of the constructible hierarchy.

LEMMA 4.2. Let $P(x, y)$ be a formula of $\mathscr{L}(\mathscr{T})$ whose only free variables are $x$ and $y$. Then there exists an $\mathscr{M}$-definable function $f$ such that for all $\alpha \in \mathscr{M}$ with probability 1:

$$
\left(x^{\alpha}\right)(E y) P\left(x^{\alpha}, y\right) \leftrightarrow\left(x^{\alpha}\right)\left(E y^{f(\alpha)}\right) P\left(x^{\alpha}, y^{f(\alpha)}\right) .
$$

Proof. Let $g(\alpha, \beta)=p\left(\left(x^{\alpha}\right)\left(E y^{\beta}\right) P\left(x^{\alpha}, y^{\beta}\right)\right) ; g$ is $\mathscr{M}$-definable by 3.1. For each $\alpha$, $\lambda \beta \mid g(\alpha, \beta)$ is a nondecreasing $\mathscr{M}$-definable function from the ordinals into the unit interval. Since the replacement axiom holds in $\mathscr{M}$, there must exist $\mathscr{M}$-definable functions $h(\alpha)$ and $f(\alpha)$ such that $h(\alpha)$ is the least upper bound of $\{g(\alpha, \beta) \mid \beta \in \mathscr{M}\}$ and $g(\alpha, f(\alpha))=h(\alpha)$.

LEMMA 4.3. Let $\mathscr{F}$ be an instance of the replacement axiom. Then $p(\mathscr{F})=1$.

Proof. The replacement axiom for $\mathscr{M}(T)$ can be split as follows: (i) the range of a function restricted to a set is contained in a set; (ii) let $P(x)$ be a formula of $\mathscr{L}(\mathscr{T})$ whose only free variable is $x$; then

$$
(y)(E z)(x)[x \in y \& P(x) \leftrightarrow x \in z] .
$$

By 4.2, (i) holds in $\mathscr{M}(T)$ for almost all $T$. But (ii) follows from (i) in $\mathscr{M}(T)$ by standard arguments implicit in Gödel $[8,9]$. Let $y \in \mathscr{M}(T)$. Then it follows from (i) by means of a Skolem-Löwenheim argument that there exists a $v \in \mathscr{M}(T)$ such that $y \in v, y \subseteq v$, and in $\mathscr{M}(T)$,

$$
(x)\left(x \in y \& P(x) \leftrightarrow x \in y \& P^{v}(x)\right),
$$

where $P^{v}(x)$ is the result of restricting the quantifiers of $P$ to $v$. Then $z=\hat{x}(x \in y \&$ $\left.P^{v}(x)\right)$ is a first-order definable subset of $v$; and $z \in \mathscr{M}(T)$, since every first-order definable subset of a member of $\mathscr{M}(T)$ is a member of $\mathscr{M}(T)$.

Proposition 4.4. Let $\left\{\mathscr{F}_{n} \mid n \in \omega\right\}$ be a sequence of $\mathscr{M}$ of sentences of $\mathscr{L}(\mathscr{T})$ of countable (in the sense of $\mathscr{M}$ ) ordinal rank. Then there exists a sentence $\mathscr{F}$ of countable ordinal rank such that for all $T$,

$$
\mathscr{M}(T) \vDash \mathscr{F} \leftrightarrow(E n)\left(\mathscr{M}(T) \vDash \mathscr{F}_{n}\right) .
$$

In addition, $\mathscr{F}$ regarded as a function of $\left\{\mathscr{F}_{n} \mid n \in \omega\right\}$, is $\mathscr{M}$-definable.

Proof. Let $\alpha$ be a countable (in the sense of $\mathscr{M}$ ) ordinal such that $o\left(\mathscr{F}_{n}\right)<\alpha$ for all $n$. Then

$$
\mathscr{M}(T) \vDash \mathscr{F}_{n} \leftrightarrow \mathscr{M}_{\alpha}(T) \vDash \mathscr{F}_{n}
$$

for all $n$ and all $T$. By standard arguments there exist a countable ordinal $\beta>\alpha$ and a formula $P\left(x^{0}\right)$ of $\mathscr{L}(\mathscr{T})$ of ordinal rank $\beta$ such that for all $n$ and all $T$,

$$
\mathscr{M}_{\alpha}(T) \vDash \mathscr{F}_{n} \leftrightarrow \mathscr{M}_{\beta}(T) \vDash P(\bar{n}) .
$$


The existence of $P\left(x^{0}\right)$ follows from two facts: (a) truth in $\mathscr{M}_{\alpha}(T)$ has a first-order definition (uniform in $T$ ) with quantifiers restricted to $\mathscr{M}_{\alpha+\omega}(T)$; (b) every firstorder definable subset of a member of $\mathscr{M}(T)$ is a member of $\mathscr{M}(T)$. The desired $\mathscr{F}$ is $\left(E x^{0}\right) P\left(x^{0}\right)$.

LEMMA 4.5. For each $n \geqq 0$, there exists an $\mathscr{M}$-definable function $\lambda \mathscr{F} \mid \mathscr{F} *$, defined for all sentences $\mathscr{F}$ of $\mathscr{L}(\mathscr{T})$ having at most $n$ unranked quantifiers, such that $p\left(\mathscr{F} \leftrightarrow \mathscr{F}^{*}\right)=1$ and $\mathscr{F}^{*}$ has countable (in the sense of $\mathscr{M}$ ) ordinal rank.

Proof. Similar to 4.1. We consider $n=0$ only, assume $\mathscr{F}$ is in prenex normal form, and define $\mathscr{F} *$ by induction on the rank of $\mathscr{F}$. If $\mathscr{F}$ has countable ordinal rank, then $\mathscr{F} *$ is $\mathscr{F}$. Let $\mathscr{F}$ have uncountable ordinal rank. Suppose $\mathscr{F}$ is $\left(E x^{\alpha}\right) \mathscr{F}_{1}\left(x^{\alpha}\right)$. Then

$$
p(\mathscr{F})=\operatorname{lub}\left\{p\left(\bigvee_{i \leqq n} \mathscr{F}_{1}\left(c_{i}\right)\right) \mid c_{i}(i \leqq n) \in C(\alpha)\right\}
$$

where lub denotes "least upper bound". As in $4.1, \bigvee_{i \leqq n} \mathscr{F}_{1}\left(c_{i}\right)$ is equivalent to a prenex normal sentence of lower rank than $\left(E x^{\alpha}\right) \mathscr{F}_{1}\left(x^{\alpha}\right)$. Then

$$
p\left(\bigvee_{i \leqq n} \mathscr{F}_{1}\left(c_{i}\right) \leftrightarrow\left(\bigvee_{i \leqq n} \mathscr{F}_{1}\left(c_{i}\right)\right)^{*}\right)=1,
$$

where $\left(\bigvee_{i \leqq n} \mathscr{F}_{1}\left(c_{i}\right)\right)^{*}$ has countable ordinal rank. It follows from the $\mathscr{M}$-definability of $\lambda \mathscr{F} \mid p(\mathscr{F})(4.1)$ that there is an $\mathscr{M}$-definable sequence $\left\{c_{i} \mid i<\omega\right\} \subseteq C(\alpha)$ such that

$$
p(\mathscr{F})=\operatorname{lub}\left\{p\left(\bigvee_{i \leqq n} \mathscr{F}_{1}\left(c_{i}\right)\right) \mid i<\omega\right\}
$$

Let $\mathscr{F}^{*}$ be equivalent to $\bigvee_{n<\omega}\left(\bigvee_{i \leqq n} \mathscr{F}\left(c_{i}\right)\right)^{*}$. $\mathscr{F} *$ exists by 4.4 .

If $\mathscr{F}$ is $\left(x^{\alpha}\right) \mathscr{F}_{1}\left(x^{\alpha}\right)$, we proceed in a manner dual to the above.

If $\mathscr{F}$ has no quantifier prefix, then $\mathscr{F}$ is equivalent, as we saw in 4.1 , to a sentence of lower ordinal rank.

Lemma 4.6. Let $\alpha$ be a cardinal of $\mathscr{M}$. Then $p(\alpha$ is a cardinal of $\mathscr{M}(T))=1$.

Proof. We sharpen the argument of 4.2. Let $\alpha$ be an uncountable cardinal of $\mathscr{M}$, and let $\beta \in \alpha$. We show $p(\beta$ can be mapped 1-1 onto $\alpha)=0$. Let $f$ be a constant $\mathscr{C}$ which denotes a function from $\beta$ into $\alpha$. For each $\gamma \in \beta$, let

$$
k(\gamma)=\{\tau \mid \tau \in \alpha \& p(f(\gamma)=\tau)>0\} .
$$

By 4.1, $\lambda \gamma \mid k(\gamma)$ is $\mathscr{M}$-definable. Since $f$ denotes a function and $p$ is countably additive in the sense of $\mathscr{M}$, it follows that $k(\gamma)$ is a countable set of $\mathscr{M}$ for all $\gamma \in \beta$. But then $\bigcup\{k(\gamma) \mid \gamma \in \beta\}$ is a subset of $\alpha$ of cardinality (in the sense of $\mathscr{M}$ ) less than that of $\alpha$, and consequently,

$$
p(\{f(\gamma) \mid \gamma \in \beta\} \subseteq \bigcup\{k(\gamma) \mid \gamma \in \beta\} \neq \alpha)=1 .
$$

Lemma 4.7. With probability 1: the power set axiom holds in $\mathscr{M}(T)$.

Proof. Our argument is related to the one given by Gödel $[9$, p. 46] to show that 
the power set axiom holds in $L$. Let $d$ be a constant of $\mathscr{C}$. It will suffice to find an ordinal $\gamma$ of $\mathscr{M}$ such that for all $c \in \mathscr{C}$,

$$
p\left(c \subseteq d \rightarrow c \in \mathscr{M}_{\gamma}(T)\right)=1 .
$$

Let $d \in \mathscr{C}(\alpha+1)-\mathscr{C}(\alpha)$. Then the members of the set denoted by $d$ can be denoted by constants of $\mathscr{C}(\alpha)$. By 4.5 there is an $\mathscr{M}$-definable function $\lambda b c \mid(b \in c)^{*}$ such that

$$
p\left(b \in c \leftrightarrow(b \in c)^{*}\right)=1
$$

for all $b, c \in \mathscr{C}$, and such that $(b \in c)^{*}$ has countable ordinal rank. For each $c \in \mathscr{C}$, let $k(c)=\lambda b \mid(b \in c)^{*}$, where $b$ is restricted to $\mathscr{C}(\alpha)$. If $k\left(c_{1}\right)=k\left(c_{2}\right)$, then

$$
p\left(c_{1} \subseteq d \& c_{2} \subseteq d \rightarrow c_{1}=c_{2}\right)=1 .
$$

Since $\mathscr{C}(\alpha)$ is a set of $\mathscr{M}$ and the power set axiom holds in $\mathscr{M}$, it follows $\{k(c) \mid c \in \mathscr{C}\}$ is a set of $\mathscr{M}$. Since the replacement axiom holds in $\mathscr{M}$, there must exist a $\gamma$ such that for all $c \in \mathscr{C}$,

$$
\left(E c^{\prime}\right)\left[c^{\prime} \in C(\gamma) \& k\left(c^{\prime}\right)=k(c)\right]
$$

LEMMA 4.8. With probability 1: the generalized continuum hypothesis holds in $\mathscr{M}(T)$.

Proof. We first sharpen the argument of 4.7, and then exploit the fact that the generalized continuum hypothesis holds in $\mathscr{M}$. Let $\alpha$ be an infinite ordinal of $\mathscr{M}$, and $\alpha^{+}$be the least ordinal greater than $\alpha$ of higher cardinality in the sense of $\mathscr{M}$. For each $c \in \mathscr{C}$, let $k(c)=\lambda b \mid(b \in c)^{*}$, where $b \in \alpha, p\left(b \in c \leftrightarrow(b \in c)^{*}\right)=1$, and $(b \in c)^{*}$ has countable rank. As in 4.7 , if $k\left(c_{1}\right)=k\left(c_{2}\right)$, then

$$
p\left(c_{1} \subseteq \alpha \& c_{2} \subseteq \alpha \rightarrow c_{1}=c_{2}\right)=1 .
$$

As in 4.7, $\{k(c) \mid c \in \mathscr{C}\}$ is a set of $\mathscr{M}$. Let $d \subseteq \mathscr{C}$ be a set of $\mathscr{M}$ such that for all $c \in \mathscr{C}$,

$$
\left(E c^{\prime}\right)\left[c^{\prime} \in d \& k\left(c^{\prime}\right)=k(c)\right]
$$

and such that

$$
\left(c_{1}\right)\left(c_{2}\right)\left[c_{1} \in d \& c_{2} \in d \rightarrow k\left(c_{1}\right) \neq k\left(c_{2}\right)\right] .
$$

Let $d^{*}$ denote the set of sets of $\mathscr{H}(T)$ denoted by the constants of $d$. Then for all $c \in \mathscr{C}$,

$$
p\left(c \subseteq \alpha \rightarrow c \in d^{*}\right)=1 .
$$

Since $k(c)$ can be regarded as a function of $\mathscr{M}$ from $\alpha$ into $\omega_{1}$, it follows that the cardinality of $d$ (in $\mathscr{M}$ ) is at most $\omega_{1}^{\alpha}=2^{\alpha}=\alpha^{+}$. But the cardinality of $d^{*}$ (in $\mathscr{M}(T)$ ) is at most that of $d$ with probability 1 .

TheOREM 4.9 (R. Solovay). With probability $1: \mathscr{M}(T)$ is a model of $Z F+G C H$ $+A C+$ "cardinals are absolute" $+V \neq L$.

Proof. Apply 4.3, 4.6, 4.7, and 4.8. For all $T, \mathscr{M}(T) \vDash \mathscr{T} \in L$ if and only if $T \in \mathscr{M}$. Clearly $p(\mathscr{T} \in L)=0$, since $\mathscr{M}$ is countable. 
We now develop Cohen's results concerning the independence of the continuum hypothesis by making purely notational changes in the proof of Theorem 4.9. Let $\gamma$ be an uncountable cardinal of $\mathscr{M}$. We study the structure $\mathscr{M}\left(T^{\gamma}\right)$, where $T^{\gamma}$ is an arbitrary subset of $\gamma$. The language $\mathscr{L}\left(\mathscr{T}^{\gamma}\right)$ is similar to the language $\mathscr{L}(\mathscr{T})$ $\left(=\mathscr{L}\left(\mathscr{T}^{\omega}\right)\right.$ ) except that $\mathscr{T}$ is replaced by $\mathscr{T}^{\gamma}$ and $\mathscr{C}(0)=\{\bar{n} \mid n \in \omega\}$ is replaced by $\{\beta \mid \beta<\gamma\} . \mathscr{M}_{\alpha}\left(T^{\gamma}\right)$ is defined in the same manner as $\mathscr{M}_{\alpha}(T)$ save that $\mathscr{M}_{0}\left(T^{\gamma}\right)=\gamma$ rather than $\omega$. Since $\gamma$ is countable, the product measure on $2^{\gamma}$ closely resembles the product measure on $2^{N}$. If $\mathscr{F}$ is a sentence of $\mathscr{L}\left(\mathscr{T}^{\gamma}\right)$, then the set

$$
\left\{T^{\gamma} \mid T^{\gamma} \in 2^{\gamma} \& \mathscr{M}\left(T^{\gamma}\right) \vDash \mathscr{F}\right\}
$$

is a Borel subset of $2^{\gamma}$; its Lebesgue measure, denoted by $p(\mathscr{F})$, is the probability that $\mathscr{F}$ is true in $\mathscr{M}\left(T^{\gamma}\right)$.

THEOREM 4.10 (R. Solovay). Let $\gamma$ be an uncountable cardinal of $\mathscr{M}$. With probability $1: \mathscr{M}\left(T^{\gamma}\right)$ is a model of $Z F+A C+$ "cardinals are absolute" $+V \neq L$. If $\gamma$ is not cofinal with $\omega$ (in the sense of $\mathscr{M}$ ), then $2^{\omega}=\gamma$ holds with probability 1 in $\mathscr{M}\left(T^{\gamma}\right)$.

Proof. Ranks are assigned to the sentences of $\mathscr{L}\left(\mathscr{T}^{\gamma}\right)$ in precisely the same way they were assigned to sentences of $\mathscr{L}(\mathscr{T})$. There is no difficulty encountered when $\mathscr{T}$ is replaced by $\mathscr{T}^{\gamma}$ in $4.1,4.2,4.3$ and $4.4 ; 4.5,4.6$, and 4.7 are unaltered by the substitution of $\mathscr{T}^{\gamma}$ for $\mathscr{T}$, since they depend only on the countable additivity of the measure. Let $g: \gamma \times \omega \rightarrow \gamma$ be the canonical 1-1 correspondence between $\gamma \times \omega$ and $\gamma$. For each $\delta>\gamma$, let $a_{\delta}$ be a constant of $\mathscr{C}$ which denotes $\left\{n \mid g(\delta, n) \in T^{\gamma}\right\}$. If $\delta_{1} \neq \delta_{2}$, then $p\left(\bar{n} \in a_{\delta_{1}} \leftrightarrow \bar{n} \in a_{\delta_{2}}\right)=\frac{1}{2}$ for each $n$, and consequently $p\left(a_{\delta_{1}}=a_{\delta_{2}}\right)=0$. It follows that $p\left(2^{\omega} \geqq \gamma\right)=1$.

Now assume $\gamma$ is not cofinal with $\omega$. The argument of 4.8 serves to show $p\left(2^{\omega}=\gamma\right)$ $=1$. For each $c \in \mathscr{C}$, let $k(c)=\lambda n \mid(\bar{n} \in c)^{*}$, where $n \in \omega$ and $(\bar{n} \in c)^{*}$ has countable ordinal rank. If $k\left(c_{1}\right)=k\left(c_{2}\right)$, then

$$
p\left(c_{1} \subseteq \omega \& c_{2} \subseteq \omega \rightarrow c_{1}=c_{2}\right)=1 .
$$

The cardinality of the set of sentences of $\mathscr{L}\left(\mathscr{T}^{\gamma}\right)$ of countable ordinal rank is $\gamma$; so $k(c)$ can be regarded as function from $\omega$ to $\gamma$. It follows $\{k(c) \mid c \in \mathscr{C}\}$ is a set (in $\mathscr{M}$ ) of cardinality at most $\gamma^{\omega}=\gamma$, since (in $\mathscr{M}$ ) $\gamma$ is not cofinal with $\omega$ and the generalized continuum hypothesis holds. But the cardinality of $2^{\omega}$ (in $\mathscr{M}\left(T^{\gamma}\right)$ ) is at most that of $\{k(c) \mid c \in \mathscr{C}\}$ with probability 1 .

Let $\left(T_{0}, T_{1}, T_{2}, \ldots\right)$ be a sequence of arbitrary subsets of $2^{N}$. We now study the effect of adjoining $\left(T_{0}, T_{1}, T_{2}, \ldots\right)$ to $\mathscr{M}$ in the same measure-theoretic fashion we studied the effect of adding an arbitrary $T$ to $\mathscr{M}$. The language $\mathscr{L}\left(\mathscr{T}_{0}, \mathscr{T}_{1}, \mathscr{T}_{2}, \ldots\right)$ is obtained by altering $\mathscr{L}(\mathscr{T})$ as follows. Replace $\mathscr{T}$ by $\mathscr{T}_{0}, \mathscr{T}_{1}, \mathscr{T}_{2}, \ldots$; add primitive symbols + (addition) and - (multiplication) with the proviso that they are to operate only on number-theoretic terms. $\mathscr{C}(0)$ is again the set of finite numerals. $\mathscr{C}(\alpha+1)$ is defined as at the beginning of $\S 4$ save that $\mathscr{T}$ is replaced by $\mathscr{T}_{0}, \mathscr{T}_{1}$, $\mathscr{T}_{2}, \ldots, \mathscr{T}_{i}, \ldots(i<\omega)$ and the formula $\phi$ can have occurrences of + and $\cdot$. Thus 
the constants of $\mathscr{C}(1)$ now denote all the subsets of $\omega$ which are arithmetical in some finite subsequence of $T_{0}, T_{1}, T_{2}, \ldots$; truth in the structure $\mathscr{M}\left(T_{0}, T_{1}, T_{2}, \ldots\right)$ and the relation " $c \in \mathscr{C} \& b \in \mathscr{M}\left(T_{0}, T_{1}, T_{2}, \ldots\right) \& c$ denotes $b$ " are defined as before.

Regard $\left(T_{0}, T_{1}, T_{2}, \ldots\right)$ as a point in the space $\left(2^{N}\right)^{N}$, and give $\left(2^{N}\right)^{N}$ the product measure induced by the measure $m$ on each factor $2 .\left(m(\{0\})=m(\{1\})=\frac{1}{2}\right.$.) Then for each sentence $\mathscr{F}$ of $\mathscr{L}\left(\mathscr{T}_{0}, \mathscr{T}_{1}, \mathscr{T}_{2}, \ldots\right)$, the set

$$
\left\{\left(T_{0}, T_{1}, T_{2}, \ldots\right) \mid \mathscr{M}\left(T_{0}, T_{1}, T_{2}, \ldots\right) \vDash \mathscr{F}\right\}
$$

is a Borel subset of $\left(2^{N}\right)^{N}$; the measure of this set, denoted by $p(\mathscr{F})$, is the probability that $\mathscr{F}$ is true in $\mathscr{M}\left(T_{0}, T_{1}, T_{2}, \ldots\right)$. The argument of 4.1 now shows: for each $n \geqq 0$, the function $p(\mathscr{F})$ restricted to sentences $\mathscr{F}$ of $\mathscr{L}\left(\mathscr{T}_{0}, \mathscr{T}_{1}, \mathscr{T}_{2}, \ldots\right)$, is $\mathscr{M}$-definable. The arguments of $4.2,4.3,4.4,4.5,4.6$, and 4.7 still work, and together they show: with probability $1, \mathscr{M}\left(T_{0}, T_{1}, T_{2}, \ldots\right)$ is a model of $\mathrm{ZF}+$ "cardinals are preserved". Actually, it is necessary to restate and reprove 4.4, 4.5, and 4.7 as $4.17 .1,4.17 .2$, and 4.17.3. That task is postponed until 4.17 is established. It is instructive to consider why 4.8 now fails. The last line of the argument of 4.8 assumes the existence of an $\mathscr{M}(T)$-definable function which assigns to each set of $\mathscr{M}(T)$ a constant of $\mathscr{C}$ that denotes it; the function is obtained by assigning $\mathscr{T}$ to $T$ and then exploiting an $\mathscr{M}$-definable well-ordering of the formulas and constants of $\mathscr{L}(\mathscr{T})$. In the present case, the language $\mathscr{L}\left(\mathscr{T}_{0}, \mathscr{T}_{1}, \mathscr{T}_{2}, \ldots\right)$ has an $\mathscr{M}$-definable well-ordering, but with probability 1 (as we shall see below) there is no $\mathscr{M}\left(T_{0}, T_{1}, T_{2}, \ldots\right)$-definable function which assigns $\mathscr{T}_{i}$ to $T_{i}$ for all $i<\omega$.

$\mathscr{M}\left(T_{0}, T_{1}, \ldots\right)$ has certain symmetries studied by Cohen [1], Feferman [2], and Solovay [29], [30] which we shall study in terms of measure-preserving transformations of $\left(2^{N}\right)^{N}$. Let $r$ be a function whose domain is $\left\{\mathscr{T}_{i} \mid i \in \omega\right\}$ and whose range is a subset of $\mathscr{C}(1) ; r$ can be extended to a function from $\mathscr{C}$ into $\mathscr{C}$ as follows:

$$
r\left(c\left(\mathscr{T}_{0}, \ldots, \mathscr{T}_{n}\right)\right)=c\left(r\left(\mathscr{T}_{0}\right), \ldots, r\left(\mathscr{T}_{n}\right)\right) ;
$$

in the same manner, $r$ can be extended to a function from the sentences of $\mathscr{L}\left(\mathscr{T}_{0}, \mathscr{T}_{1}, \ldots\right)$ into itself. If $r$ is a map from $\mathscr{C}(1)$ into $\mathscr{C}(1)$, then $r$ induces a map $r^{*}$ from $\left(2^{N}\right)^{N}$ into $\left(2^{N}\right)^{N}$ as follows:

$$
\begin{gathered}
n \in r^{*}\left(T_{i}\right) \leftrightarrow \mathscr{M}\left(T_{0}, T_{1}, \ldots\right) \vDash \bar{n} \in r\left(\mathscr{T}_{i}\right) ; \\
r^{*}\left(T_{0}, T_{1}, \ldots\right)=\left(r^{*}\left(T_{0}\right), r^{*}\left(T_{1}\right), \ldots\right) .
\end{gathered}
$$

We say $r$ is a map from $\mathscr{C}(1)$ onto $\mathscr{C}(1)$ if $r$ is a map from $\mathscr{C}(1)$ into $\mathscr{C}(1)$ and if for each $i \in \omega$, there is a $u_{i} \in \mathscr{C}(1)$ such that $\left.r\left(u_{i}\right)=\mathscr{T}_{i}{ }^{\left({ }^{11}\right.}\right)$. If $r$ is a map from $\mathscr{C}(1)$ onto $\mathscr{C}(1)$, then $r^{*}$ is a $1-1$ map from $\left(2^{N}\right)^{N}$ into $\left(2^{N}\right)^{N}$.

LeMmA 4.11. Let $r$ be a map of $\mathscr{C}(1)$ onto $\mathscr{C}(1)$. Then $\mathscr{M}\left(T_{0}, T_{1}, \ldots\right) \vDash r(\mathscr{F})$ $\leftrightarrow \mathscr{M}\left(r^{*}\left(T_{0}\right), r^{*}\left(T_{1}\right), \ldots\right) \vDash \mathscr{F}$, for all $\left(T_{0}, T_{1}, \ldots\right)$.

${ }^{(11)}$ Let $c, d \in \mathscr{C}$. We say $c=d$ if $c$ and $d$ denote the same member of $\mathscr{M}\left(T_{0}, T_{1}, T_{2}, \ldots\right)$ for all $\left(T_{0}, T_{1}, T_{2}, \ldots\right)$. J. Rosenthal [21] has shown that this notion of equality is $\mathscr{M}$-definable. His result is not needed in the present paper. 
Proof. By induction on the rank of $\mathscr{F}$. (We use the same notion of rank employed in 4.1.) It is sufficient to consider two cases.

Case 1. $\mathscr{F}$ is $\bar{n} \in \mathscr{T}_{i}$. Then $\mathscr{M}\left(T_{0}, T_{1}, \ldots\right) \vDash r\left(\bar{n} \in \mathscr{T}_{i}\right) \leftrightarrow \mathscr{M}\left(T_{0}, T_{1}, \ldots\right) \vDash \bar{n}$ $\in r\left(\mathscr{T}_{i}\right) \leftrightarrow n \in r^{*}\left(T_{i}\right) \leftrightarrow \mathscr{M}\left(r\left(T_{0}\right), r\left(T_{1}\right), \ldots\right) \vDash \bar{n} \in \mathscr{T}_{i}$.

Case 2. $\mathscr{F}$ is $\left(E x^{\alpha}\right) \mathscr{G}\left(x^{\alpha}, b\right)$, where $b$ is the only member of $\mathscr{C}$ occurring in $\mathscr{F}$. It is easily checked that $r$ maps $\mathscr{C}(\alpha)$ onto $\mathscr{C}(\alpha)$; i.e., for each $d \in \mathscr{C}(\alpha)$, there is a $c \in \mathscr{C}(\alpha)$ such that $r(c)=d$. The following formulas are equivalent:

$$
\begin{aligned}
& \mathscr{M}\left(r^{*}\left(T_{0}\right), r^{*}\left(T_{1}\right), \ldots\right) \vDash\left(E x^{\alpha}\right) \mathscr{G}\left(x^{\alpha}, b\right) ; \\
& (E c)\left[c \in \mathscr{C}(\alpha) \& \mathscr{M}\left(r^{*}\left(T_{0}\right), r^{*}\left(T_{1}\right), \ldots\right) \vDash \mathscr{G}(c, b)\right] ; \\
& (E c)\left[c \in \mathscr{C}(\alpha) \& \mathscr{M}\left(T_{0}, T_{1}, \ldots\right) \vDash \mathscr{G}(r(c), r(b))\right] ; \\
& (E d)\left[d \in \mathscr{C}(\alpha) \& \mathscr{M}\left(T_{0}, T_{1}, \ldots\right) \vDash \mathscr{G}(d, r(b))\right] ; \\
& \mathscr{M}\left(T_{0}, T_{1}, \ldots\right) \vDash r\left(\left(E x^{\alpha}\right) \mathscr{G}\left(x^{\alpha}, b\right)\right) .
\end{aligned}
$$

The second formula is equivalent to the third by the induction hypothesis.

LEMMA 4.12. Let $r$ be a map of $\mathscr{C}(1)$ onto $\mathscr{C}(1)$ such that the induced map $r^{*}:\left(2^{N}\right)^{N}$ $\rightarrow\left(2^{N}\right)^{N}$ is measure-preserving. Then $p(\mathscr{F})=p(r(\mathscr{F}))$.

Proof. $r^{*}$ is $1-1$ and maps $\left(2^{N}\right)^{N}$ onto a set whose complement has measure 0. Now apply 4.11 .

Feferman's transformation lemma $[3,4.8]$ is a forcing-theoretic version of our Lemma 4.12.

Let $\mathscr{C}\left(\alpha ; \mathscr{T}_{0}, \mathscr{T}_{1}, \ldots, \mathscr{T}_{k-1}\right)(k \geqq 0)$ be the set of all constants of $\mathscr{C}(\alpha)$ whose $\mathscr{T}_{i}$-symbols are included in the sequence $\mathscr{T}_{0}, \ldots, \mathscr{T}_{k-1}$. Let $\mathscr{M}\left(\alpha ; T_{0}, \ldots, T_{k-1}\right)$ be the set of all sets of $\mathscr{M}_{\alpha}\left(T_{0}, T_{1}, T_{2}, \ldots\right)$ denoted by members of

$$
\mathscr{C}\left(\alpha ; \mathscr{T}_{0}, \ldots, \mathscr{T}_{k-1}\right) \text {. }
$$

It is clear that $\mathscr{C}$ has a constant which denotes $\mathscr{M}\left(\alpha ; T_{0}, \ldots, T_{k-1}\right)$; let us adopt the convention of denoting $\mathscr{M}\left(\alpha ; T_{0}, \ldots, T_{k-1}\right)$ by $\mathscr{M}\left(\alpha ; \mathscr{T}_{0}, \ldots, \mathscr{T}_{k-1}\right)$. Similarly, let

$$
\mathscr{C}\left(\mathscr{T}_{0}, \ldots, \mathscr{T}_{k-1}\right)=\bigcup\left\{\mathscr{C}\left(\alpha ; \mathscr{T}_{0}, \ldots, \mathscr{T}_{k-1}\right) \mid \alpha \in \mathscr{M}\right\},
$$

and let $\mathscr{M}\left(\mathscr{T}_{0}, \ldots, \mathscr{T}_{k-1}\right)$ denote $\bigcup\left\{\mathscr{M}\left(\alpha ; T_{0}, \ldots, T_{k-1}\right) \mid \alpha \in \mathscr{M}\right\}$.

Proposition 4.13 (R. Solovay). For each $k \geqq 0: \mathscr{M}\left(\mathscr{T}_{0}, \ldots, \mathscr{T}_{k-1}\right)$ is well-ordered with probability 1.

Proof. Let $\mathscr{N}=\mathscr{M}\left(T_{0}, T_{1}, T_{2}, \ldots\right)$ be a model of $\mathrm{ZF}$ with the allowable exception of the power set axiom. Fix $k \geqq 0$. In $\mathscr{N}$ we can define truth in $\mathscr{N}$ for all ranked sentences whose $\mathscr{T}_{i}$-symbols are included in the list $\mathscr{T}_{0}, \ldots, \mathscr{T}_{k-1}$. It follows by standard arguments that there exists a 1-1 $\mathscr{N}$-definfible map $h_{k}$ of $\mathscr{M}\left(T_{0}, \ldots, T_{k-1}\right)$ into $\mathscr{C}\left(\mathscr{T}_{0}, \ldots, \mathscr{T}_{k-1}\right)$, i.e., each set of $\mathscr{M}\left(T_{0}, \ldots, T_{k-1}\right)$ is mapped to a constant of $\mathscr{C}\left(\mathscr{T}_{0}, \ldots, \mathscr{T}_{k-1}\right)$ which denotes that set. But there exists an $\mathscr{M}$-definable (hence $\mathscr{N}$-definable) well-ordering of $\mathscr{C}$. The definition of $h_{k}$ has $T_{0}, \ldots, T_{k-1}$ as parameters. 
LEMma 4.14 (R. Solovay). Let $\mathscr{F}\left(x^{\alpha}\right)$ be a formula of $\mathscr{L}\left(\mathscr{T}_{0}, \mathscr{T}_{1}, \ldots\right)$ whose only free variable is $x^{\alpha}$, and whose $\mathscr{T}_{i}$-symbols are included among $\mathscr{T}_{0}, \ldots, \mathscr{T}_{k-1}$. Then with probability 1 ,

$$
\left(E x^{\alpha}\right) \mathscr{F}\left(x^{\alpha}\right) \leftrightarrow\left(E x^{\alpha}\right)\left[\mathscr{F}\left(x^{\alpha}\right) \& x^{\alpha} \in \mathscr{M}\left(\alpha ; \mathscr{T}_{0}, \ldots, \mathscr{T}_{k}\right)\right] .
$$

Similarly, with probability 1 ,

$$
(E x) \mathscr{F}(x) \leftrightarrow(E x)\left[\mathscr{F}(x) \& x \in \mathscr{M}\left(\mathscr{T}_{0}, \ldots, \mathscr{T}_{k}\right)\right] .
$$

Proof. Let $k=1$. To prove the first half of the lemma, it is enough to prove: for each sequence $c_{0}, c_{1}, \ldots, c_{n} \in \mathscr{C}(\alpha)$, there exists a sequence $r\left(c_{0}\right), r\left(c_{1}\right), \ldots, r\left(c_{n}\right)$ $\in \mathscr{C}\left(\alpha ; \mathscr{T}_{0}, \mathscr{T}_{1}\right)$ such that

$$
p\left(\bigvee_{i \leqq n} \mathscr{F}\left(c_{i}\right)\right)=p\left(\bigvee_{i \leqq n} \mathscr{F}\left(r\left(c_{i}\right)\right)\right)
$$

Let $m$ be so large that $c_{0}, c_{1}, \ldots, c_{n} \in \mathscr{C}\left(\alpha ; \mathscr{T}_{0}, \ldots, \mathscr{T}_{m}\right)$. Consider the following map $r$ of $\mathscr{C}(1)$ into $\mathscr{C}(1)$ :

$$
\begin{aligned}
r\left(\mathscr{T}_{0}\right) & =\mathscr{T}_{0} ; r\left(\mathscr{T}_{i+m+1}\right)=\mathscr{T}_{i+2} \quad(i \geqq 0) ; \\
r\left(\mathscr{T}_{j+1}\right) & =\hat{x}_{0}\left(\bar{m} x_{0}+j \in \mathscr{T}_{1}\right) \quad(j<m) .
\end{aligned}
$$

It is not difficult to check that $r$ maps $\mathscr{C}(1)$ onto $\mathscr{C}(1)$ and that $r^{*}$ is measurepreserving $\left({ }^{12}\right)$. Clearly, $r\left(c_{i}\right) \in \mathscr{C}\left(\alpha ; \mathscr{T}_{0}, \mathscr{T}_{1}\right)$ and $r\left(\mathscr{F}\left(c_{i}\right)\right)$ is $\mathscr{F}\left(r\left(c_{i}\right)\right)$ for each $i \leqq n$. Now apply 4.12.

We specify some maps of $\mathscr{C}(1)$ into $\mathscr{C}(1)$ needed for the proof of Lemma 4.15. For each $n \geqq 0$ and $i \geqq 0$, let

$$
\begin{aligned}
\left(\mathscr{T}_{m}\right)_{i} & =\hat{x}_{0}\left[\left(2^{i+1} x^{0}+2^{i}-1\right) \in \mathscr{T}_{m}\right] ; \\
\left(\mathscr{T}_{m}\right)^{n} & =\hat{x}_{0}\left[\left(2^{n} x^{0}+2^{n}-1\right) \in \mathscr{T}_{m}\right] .
\end{aligned}
$$

For each $m, n \geqq 0$, we define a map $r_{n}^{m}$ of $\mathscr{C}(1)$ into $\mathscr{C}(1)$ :

$$
\begin{aligned}
r_{n}^{m}\left(\mathscr{T}_{j}\right) & =\mathscr{T}_{j} & & (j<m) ; \\
r_{n}^{m}\left(\mathscr{T}_{m+i}\right) & =\left(\mathscr{T}_{m}\right)_{i} & & (i<n) ; \\
r_{n}^{m}\left(\mathscr{T}_{m+n}\right) & =\left(\mathscr{T}_{m}\right)^{n} ; & & \\
r_{n}^{m}\left(\mathscr{T}_{m+n+k}\right) & =\mathscr{T}_{m+k} & & (k \geqq 1) .
\end{aligned}
$$

It is not hard to see that $\left(r_{n}^{m}\right)^{*}:\left(2^{N}\right)^{N} \rightarrow\left(2^{N}\right)^{N}$ is measure-preserving (see footnote $\left({ }^{12}\right)$ ). We claim that $r_{n}^{m}$ maps $\mathscr{C}(1)$ onto $\mathscr{C}(1)$. Consider the constant $d_{n}^{m} \in \mathscr{C}(1)$ defined by

$$
\begin{aligned}
x^{0} \in d_{n}^{m} \leftrightarrow & \left(E y^{0}\right)\left(y^{0} \in \mathscr{T}_{m+n} \& x^{0}=2^{n} y^{0}+2^{n}-1\right) \\
& \vee(E i)_{0 \leqq i<n}\left(E y^{0}\right)\left(y^{0} \in \mathscr{T}_{m+i} \& x^{0}=2^{i+1} y^{0}+2^{i}-1\right) .
\end{aligned}
$$

$\left({ }^{12}\right)$ Cf. Halmos [10, p. 159 (7)]. 
Clearly, $r_{n}^{m}\left(d_{n}^{m}\right)=\mathscr{T}_{m}$. Thus $r_{n}^{m}$ is a map of $\mathscr{C}(1)$ into $\mathscr{C}(1)$ which meets the hypotheses of Lemma 4.12.

Lemma 4.15 (R. Solovay). With probability 1, the dependent axiom of choice holds in $\mathscr{M}\left(T_{0}, T_{1}, T_{2}, \ldots\right)$.

Proof. Let $\mathscr{F}(x, y)$ be a formula of $\mathscr{L}\left(\mathscr{T}_{0}, \mathscr{T}_{1}, \ldots\right)$ whose only free variables are $x$ and $y$ and all of whose $\mathscr{T}_{i}$-symbols are included among $\mathscr{T}_{0}, \mathscr{T}_{1}, \ldots, \mathscr{T}_{m-1}(m \geqq 0)$. We intend to find an $f \in \mathscr{C}\left(\mathscr{T}_{0}, \ldots, \mathscr{T}_{m}\right)$ such that $f$ denotes a function on $\omega$ and such that with probability 1 ,

$$
(x)(E y) \mathscr{F}(x, y) \rightarrow\left(x^{0}\right) \mathscr{F}\left(f\left(x^{0}\right), f\left(x^{0}+\overline{1}\right)\right) .
$$

For the sake of notational simplicity, let $m=1$. By 4.14 , for each $n \geqq 0$, the following two sentences are equivalent with probability 1 :

$$
\begin{aligned}
& (x)(E y)\left[x \in \mathscr{M}\left(\mathscr{T}_{0}, \ldots, \mathscr{T}_{n}\right) \rightarrow \mathscr{F}(x, y)\right] \\
& (x)(E y)\left[x \in \mathscr{M}\left(\mathscr{T}_{0}, \ldots, \mathscr{T}_{n}\right) \rightarrow \mathscr{F}(x, y) \& y \in \mathscr{M}\left(\mathscr{T}_{0}, \ldots, \mathscr{T}_{n+1}\right)\right] .
\end{aligned}
$$

With the aid of 4.12 , we apply $r_{n+1}^{1}$ to the above pair of sentences to obtain another pair equivalent with probability 1 :

$(x)(E y)\left[x \in \mathscr{M}\left(\mathscr{T}_{0},\left(\mathscr{T}_{1}\right)_{0}, \ldots,\left(\mathscr{T}_{1}\right)_{n-1}\right) \rightarrow \mathscr{F}(x, y)\right] ;$

$(x)(E y)\left[x \in \mathscr{M}\left(\mathscr{T}_{0},\left(\mathscr{T}_{1}\right)_{0}, \ldots,\left(\mathscr{T}_{1}\right)_{n-1}\right) \rightarrow \mathscr{F}(x, y) \& y \in \mathscr{M}\left(\mathscr{T}_{0},\left(\mathscr{T}_{1}\right)_{0}, \ldots,\left(\mathscr{T}_{1}\right)_{n}\right)\right]$

(Note: $\mathscr{M}\left(\mathscr{T}_{0},\left(\mathscr{T}_{1}\right)_{0}, \ldots,\left(\mathscr{T}_{1}\right)_{n}\right)$ denotes the collection of all sets of $\mathscr{M}\left(T_{0}, T_{1}\right.$, $\left.T_{2}, \ldots\right)$ denoted by constants of the form $r_{n+1}^{1}(c)$ for some $c \in \mathscr{C}\left(\mathscr{T}_{0}, \mathscr{T}_{1}, \ldots, \mathscr{T}_{n+1}\right)$.) It follows from 4.13 that the sequence of sets $\mathscr{M}\left(\mathscr{T}_{0},\left(\mathscr{T}_{1}\right)_{0}, \ldots,\left(\mathscr{T}_{1}\right)_{n}\right)(n \geqq 0)$ can be well-ordered uniformly in $n$ with probability 1 . But then for some $f \in \mathscr{C}$, we have with probability $1:$ if $(x)(E y) \mathscr{F}(x, y)$, then

$$
\begin{aligned}
f(\overline{0}) & \in \mathscr{M}\left(\mathscr{T}_{0}\right), \\
f(\bar{n}+\overline{1}) & \in \mathscr{M}\left(\mathscr{T}_{0},\left(\mathscr{T}_{1}\right)_{0}, \ldots,\left(\mathscr{T}_{1}\right)_{n}\right) \quad(n \geqq 0), \\
& \left(x^{0}\right) \mathscr{F}\left(f\left(x^{0}\right), f\left(x^{0}+\overline{1}\right)\right) .
\end{aligned}
$$

We can insist that $f \in \mathscr{C}\left(\mathscr{T}_{0}, \mathscr{T}_{1}\right)$, since the constants that denote the well-orderings needed in the definition of $f$ are members of $\mathscr{C}\left(\mathscr{T}_{0}, \mathscr{T}_{1}\right)$.

The argument of Lemma 4.15 can be greatly simplified, if one merely wishes to establish the countable axiom of choice (see footnote $\left({ }^{2}\right)$ ). In fact, Proposition 4.13 and Lemma 4.14 readily imply: with probability 1 , the product of a family of nonempty sets, indexed by a well-ordered set, is nonempty.

A very simple notion of conditional probability of truth in $\mathscr{M}\left(T_{0}, T_{1}, T_{2}, \ldots\right)$ will make it possible to strengthen Lemma 4.12. Let $\left\{\mathscr{T}_{i} \mid i \in K\right\}$ be a finite set of $\mathscr{T}_{i}$-symbols; let $A_{i} \subseteq \omega$ for each $i \in K$. The conditional probability that $\mathscr{F}$ is true in $\mathscr{M}\left(T_{0}, T_{1}, T_{2}, \ldots\right)$, given that $T_{i}=A_{i}$ for all $i \in K$, is denoted by

$$
p\left(\mathscr{F} \mid \mathscr{T}_{i}=A_{i}, i \in K\right)
$$


and is defined as follows. Let $\left(2^{N}\right)_{K}^{N}$ be the set of all sequences $\left(T_{0}, T_{1}, T_{2}, \ldots\right)$ such that $T_{i}=A_{i}$ for all $i \in K$. Think of $\left(2^{N}\right)_{K}^{N}$ as the product of countably many copies of $2^{N}$ and finitely many one-point spaces, one one-point space $\left\{A_{i}\right\}$ for each $i \in K$. Give each copy of $2^{N}$ the usual Lebesgue measure, and give each one-point space measure 1. Let $\left(2^{N}\right)_{K}^{N}$ have the product measure induced by the measures assigned to its factors. Then $p\left(\mathscr{F} \mid \mathscr{T}_{i}=A_{i}, i \in K\right)$ is the measure of the set of all sequences $\left(T_{0}, T_{1}, T_{2}, \ldots\right)$ of $\left(2^{N}\right)_{K}^{N}$ such that $\mathscr{F}$ is true in $\mathscr{M}\left(T_{0}, T_{1}, T_{2}, \ldots\right)$.

Let $r$ be a map of $\mathscr{C}(1)$ into $\mathscr{C}(1)$. Then for each $i, r\left(\mathscr{T}_{i}\right)$ is a member of $\mathscr{C}(1)$ in which only finitely many $\mathscr{T}_{j}$-symbols occur. Suppose for each $i$, either all or none of the $\mathscr{T}_{j}$-symbols occurring in $r\left(\mathscr{T}_{i}\right)$ also occur in the set $\left\{\mathscr{T}_{i} \mid i \in K\right\}$. We describe this state of affairs by saying that fixing $\left\{\mathscr{T}_{i} \mid i \in K\right\}$ has the effect of fixing $\left\{r\left(\mathscr{T}_{i}\right) \mid i \in K_{r}\right\}$, where $K_{r}$ is the set of all $i$ such that every $\mathscr{T}_{j}$-symbol occurring in $r\left(\mathscr{T}_{i}\right)$ also occurs in $\left\{\mathscr{T}_{i} \mid i \in K\right\}$. We will always assume, for convenience only, that $K$ and $K_{r}$ are finite. Observe that fixing $T_{i}=A_{i}$ for all $i \in K$ has the effect of fixing $r^{*}\left(T_{i}\right)=B_{i}$ for all $i \in K_{r}$, where $B_{i}$ is the set in $\mathscr{M}\left(T_{0}, T_{1}, T_{2}, \ldots\right)$ denoted by $r\left(\mathscr{T}_{i}\right)$ when $T_{i}=A_{i}$ for all $i \in K$.

LEMMA 4.16. Let $r$ be a map of $\mathscr{C}(1)$ onto $\mathscr{C}(1)$ such that $r^{*}:\left(2^{N}\right)^{N} \rightarrow\left(2^{N}\right)^{N}$ is measure-preserving. Suppose that fixing $\left\{\mathscr{T}_{i} \mid i \in K\right\}$ has the effect of fixing

$$
\left\{r\left(\mathscr{T}_{i}\right) \mid i \in K_{r}\right\} .
$$

Then for each sentence $\mathscr{F}$ of $\mathscr{L}\left(\mathscr{T}_{0}, \mathscr{T}_{1}, \mathscr{T}_{2}, \ldots\right)$,

$$
p\left(r(\mathscr{F}) \mid \mathscr{T}_{i}=A_{i}, i \in K\right)=p\left(\mathscr{F} \mid \mathscr{T}_{i}=B_{i}, i \in K_{r}\right)
$$

where for each $i \in K_{r}, B_{i}$ is the set denoted by $r\left(\mathscr{T}_{i}\right)$ when $T_{i}=A_{i}$ for all $i \in K$.

Proof. We extend the argument of 4.12. The map $r^{*}:\left(2^{N}\right)^{N} \rightarrow\left(2^{N}\right)^{N}$ is $1-1$. Let $r_{K}^{*}$ be the restriction of $r^{*}$ to $\left(2^{N}\right)_{K}^{N}$. Then $r_{K}^{*}$ is a $1-1$ map of $\left(2^{N}\right)_{K}^{N}$ into $\left(2^{N}\right)_{K_{r}}^{N}$. The lemma will now follow from 4.11, after we show $r_{K}^{*}$ is measure-preserving. Let $\left\{c_{i} \mid i \in \omega\right\}$ be a sequence of constants of $\mathscr{C}$ which denote subsets of $\omega$. We say $\left\{c_{i} \mid i \leqq n\right\}$ is a sequence of independent, uniformly distributed $\omega$-terms if

$$
p\left(\bar{m}_{1} \in c_{i 1} \& \bar{m}_{2} \in c_{i 2} \& \cdots \& \bar{m}_{k} \in c_{i k}\right)=1 / 2^{k}
$$

whenever $0 \leqq i 1 \leqq i 2 \leqq \cdots \leqq i k \leqq n$ and $m_{j}=m_{p} \rightarrow i j \neq i p$ for $1 \leqq j<p \leqq k$. We say $\left\{c_{i} \mid i \in \omega\right\}$ is a sequence of independent, uniformly distributed $\omega$-terms if $\left\{c_{i} \mid i \leqq n\right\}$ is such a sequence for all $n \geqq 0$. It follows from 4.12 that $\left\{r\left(\mathscr{T}_{i}\right) \mid i \in \omega\right\}$ is a sequence of independent, uniformly distributed $\omega$-terms. This last fact implies $r_{K}^{*}$ is measurepreserving (cf. Halmos [10, p. 191-192]).

CoROllaRY 4.17. Let $\left\{\mathscr{T}_{i} \mid i \in K\right\}$ include all the $\mathscr{T}_{i}$-symbols occurring in the sentence $\mathscr{F}$ of $\mathscr{L}\left(\mathscr{T}_{0}, \mathscr{T}_{1}, \mathscr{T}_{2}, \ldots\right)$. Then for every sequence $\left\{A_{i} \mid i \in K\right\}$ of subsets of $\omega, p\left(\mathscr{F} \mid \mathscr{T}_{i}=A_{i}, i \in K\right)=0$ or $1\left({ }^{13}\right)$.

$\left({ }^{13}\right)$ S. Kochen pointed out that Corollary 4.17 is an instance of the $0-1$ law $[10$, p. 201]. 
Proof. Let $b$ denote an arbitrary propositional combination of sentences of the form $\bar{m} \in \mathscr{T}_{i}, \bar{n} \notin \mathscr{T}_{j}$, where $i, j \notin K$. Then $p(b)$ can be thought of as the measure of an arbitrary finite union of basic open subsets of $\left(2^{N}\right)_{K}^{N} .\left(\left(2^{N}\right)_{K}^{N}\right.$ is defined just before 4.16.) It follows from 4.16 that

$$
p\left(\mathscr{F} \& b \mid \mathscr{T}_{i}=A_{i}, i \in K\right)=p(b) p\left(\mathscr{F} \mid \mathscr{T}_{i}=A_{i}, i \in K\right) .
$$

For example, if $K=\{0\}, b$ is $\bar{m} \in \mathscr{T}_{1}$, and $r$ is the map defined by $r\left(\mathscr{T}_{1}\right)=\omega-\mathscr{T}_{1}$ and $r\left(\mathscr{T}_{i}\right)=\mathscr{T}_{i}(i \neq 1)$, then

$$
p\left(\mathscr{F} \& \bar{m} \in \mathscr{T}_{1} \mid \mathscr{T}_{0}=A_{0}\right)=p\left(\mathscr{F} \& \bar{m} \notin \mathscr{T}_{1} \mid \mathscr{T}_{0}=A_{0}\right)
$$

by 4.16 , and consequently,

$$
p\left(\mathscr{F} \& m \in \mathscr{T}_{1} \mid \mathscr{T}_{0}=A_{0}\right)=\frac{1}{2} p\left(\mathscr{F} \mid \mathscr{T}_{0}=A_{0}\right) .
$$

For each $\delta>0$, there exists a $b$ such that

$$
p\left(\mathscr{F} \leftrightarrow b \mid \mathscr{T}_{i}=A_{i}, i \in K\right) \geqq 1-\delta .
$$

But then $p\left(\mathscr{F} \mid \mathscr{T}_{i}=A_{i}, i \in K\right)$ must be a solution of $x=x^{2}$.

Suppose $\left\{\mathscr{T}_{i} \mid i \in K\right\}$ includes all the $\mathscr{T}_{i}$-symbols occurring in $\mathscr{F}$, where $\mathscr{F}$ is a sentence of $\mathscr{L}\left(\mathscr{T}_{0}, \mathscr{T}_{1}, \mathscr{T}_{2}, \ldots\right)$. Then Corollary 4.17 tells us that the truth-value of $\mathscr{F}$ in the structure $\mathscr{M}\left(T_{0}, T_{1}, T_{2}, \ldots\right)$ is determined by the choice of $\left\{T_{i} \mid i \in K\right\}$ for almost all sequences $\left(T_{0}, T_{1}, T_{2}, \ldots\right)$. Let $\left(2^{N}\right)^{K}$ be the product of $K$-many copies of $2^{N}$, one copy of $2^{N}$ for each $i \in K$. Give $\left(2^{N}\right)^{K}$ the usual product measure. Identify the sequences $\left\{A_{i} \mid i \in K\right\}$ with the points of $\left(2^{N}\right)^{K}$. Then by Fubini's theorem $p(\mathscr{F})$ is the measure of the set of all sequences $\left\{A_{i} \mid i \in K\right\}$ such that $p\left(\mathscr{F} \mid \mathscr{T}_{i}=A_{i}, i \in K\right)$ $=1$.

Proposition 4.17.1 (CF. 4.4). Let $\left\{\mathscr{F}_{n} \mid n \in \omega\right\}$ be a sequence of $\mathscr{M}$ of sentences of $\mathscr{L}\left(\mathscr{T}_{0}, \mathscr{T}_{1}, \ldots\right)$ of countable (in the sense of $\left.\mathscr{M}\right)$ ordinal rank such that for some $k$, no $\mathscr{T}_{i}$-symbol with $i \geqq k$ occurs in any $\mathscr{F}_{n}$. Then there exists a sentence $\mathscr{F}$ of countable ordinal rank containing no $\mathscr{T}_{i}$-symbols not occurring in at least one $\mathscr{F}_{n}$ and such that for almost all $\left(T_{0}, T_{1}, \ldots\right)$,

$$
\mathscr{M}\left(T_{0}, T_{1}, \ldots\right) \vDash \mathscr{F} \leftrightarrow(E n)\left(\mathscr{M}\left(T_{0}, T_{1}, \ldots\right) \vDash \mathscr{F}_{n}\right) .
$$

In addition, $\mathscr{F}$ regarded as a function of $\left\{\mathscr{F}_{n} \mid n \in \omega\right\}$ is $\mathscr{M}$-definable.

Proof. The argument of 4.4 establishes the first part of the proposition, namely, that the desired $\mathscr{F}$ exists. The $\mathscr{M}$-definability of $\mathscr{F}$ as a function of $\left\{\mathscr{F}_{n} \mid n \in \omega\right\}$ follows from the $\mathscr{M}$-definability of the probability function $p$ restricted to sentences of $\mathscr{L}\left(\mathscr{T}_{0}, \mathscr{T}_{1}, \ldots\right)$ of countable ordinal rank and from the following equations which characterize $\mathscr{F}$ up to a set of measure 0 :

$$
\begin{gathered}
(i)\left[p\left(\bigvee_{n \leqq i} \mathscr{F}_{n} \rightarrow \mathscr{F}\right)=1\right] ; \\
(\delta)(E i)\left[p\left(\mathscr{F} \rightarrow \bigvee_{n \leqq i} \mathscr{F}_{n}\right) \geqq 1-\delta\right] ;
\end{gathered}
$$

$\delta$ is a variable ranging over the positive rationals. 
LEMMA 4.17.2 (CF. 4.5). For each $n \geqq 0$ : there exists an $\mathscr{M}$-definable function $\lambda \mathscr{F} \mid \mathscr{F} *$, defined for all sentences $\mathscr{F}$ of $\mathscr{L}\left(\mathscr{T}_{0}, \mathscr{T}_{1}, \ldots\right)$ having at most $n$ unranked quantifiers, such that $p\left(\mathscr{F} \leftrightarrow \mathscr{F}^{*}\right)=1$ and such that $\mathscr{F}^{*}$ has countable (in the sense of $\mathscr{M})$ ordinal rank and contains no $\mathscr{T}_{i}$-symbols not occurring in $\mathscr{F}$.

Proof. We proceed by an induction on rank after the fashion of 4.5. Let $\mathscr{F}$ be a prenex normal sentence of uncountable ordinal rank of the form $\left(E x^{\alpha}\right) \mathscr{F}_{1}\left(x^{\alpha}\right)$ such that $\mathscr{T}_{0}$ is the only $\mathscr{T}_{i}$-symbol occurring in $\mathscr{F}$. By 4.14 ,

$$
p(\mathscr{F})=\operatorname{lub}\left\{p\left(\bigvee_{i \leqq n} \mathscr{F}_{1}\left(c_{i}\right)\right) \mid c_{i} \in \mathscr{C}\left(\alpha ; \mathscr{T}_{0}, \mathscr{T}_{1}\right)\right\}
$$

where lub denotes "least upper bound". As in $4.1, \bigvee_{i \leqq n} \mathscr{F}_{1}\left(c_{i}\right)$ is equivalent to a prenex normal sentence of lower rank than $\left(E x^{\alpha}\right) \mathscr{F}_{1}\left(x^{\alpha}\right)$. Then

$$
p\left(\bigvee_{i \leqq n} \mathscr{F}_{1}\left(c_{i}\right) \leftrightarrow\left(\bigvee_{i \leqq n} \mathscr{F}_{1}\left(c_{i}\right)\right)^{*}\right)=1,
$$

where $\left(\bigvee_{i \leqq n} \mathscr{F}_{1}\left(c_{i}\right)\right)^{*}$ has countable ordinal rank and contains no $\mathscr{T}_{i}$-symbols other than $\mathscr{T}_{0}$ or $\mathscr{T}_{1}$. It follows from the $\mathscr{M}$-definability of $p$ that there exists an $\mathscr{M}$-definable sequence $\left\{c_{i} \mid i<\omega\right\} \subseteq \mathscr{C}\left(\alpha ; \mathscr{T}_{0}, \mathscr{T}_{1}\right)$ such that

$$
p(\mathscr{F})=\operatorname{lub}\left\{p\left(\bigvee_{i \leqq n} \mathscr{F}_{1}\left(c_{i}\right)\right) \mid n<\omega\right\}
$$

Let $\mathscr{G}$ be a sentence of countable ordinal rank equivalent with probability 1 to $\bigvee_{n<\omega}\left(\bigvee_{i \leqq n} \mathscr{F}_{1}\left(c_{i}\right)\right)^{*}$. The existence of $\mathscr{G}$, as well as the fact that $\mathscr{G}$ contains no $\mathscr{T}_{i}$-symbols other than $\mathscr{T}_{0}$ or $\mathscr{T}_{1}$, is a consequence of 4.17.1.

If $\mathscr{G}$ contains only $\mathscr{T}_{0}$, then the desired $\mathscr{F}^{*}$ is $\mathscr{G}$. Suppose $\mathscr{G}$ contains both $\mathscr{T}_{0}$ and $\mathscr{T}_{1}$. We know that $p(\mathscr{F} \leftrightarrow \mathscr{G})=1$ and that the only $\mathscr{T}_{i}$-symbol occurring in $\mathscr{F}$ is $\mathscr{T}_{0}$. It follows from 4.17 that $p\left(\mathscr{G} \mid \mathscr{T}_{0}=T_{0}\right)$ equals 0 or 1 for almost all $T_{0}$. The function $\lambda T_{0} \mid p\left(\mathscr{G} \mid \mathscr{T}_{0}=T_{0}\right)$ is $\mathscr{M}\left(T_{0}, T_{1}, \ldots\right)$-definable for almost all $\left(T_{0}, T_{1}, \ldots\right)$; its definition contains no $\mathscr{T}_{i}$-symbol other than $\mathscr{T}_{0}$, and has countable ordinal rank because $\mathscr{G}$ does. Thus there is a formula $\mathscr{H}\left(\mathscr{T}_{0}, x^{0}\right)$ of countable ordinal rank such that for almost all $\left(T_{0}, T_{1}, \ldots\right)$ :

$$
\begin{aligned}
& \mathscr{M}\left(T_{0}, T_{1}, \ldots\right) \vDash \mathscr{H}\left(\mathscr{T}_{0}, \overline{0}\right) \leftrightarrow p\left(\mathscr{G} \mid \mathscr{T}_{0}=T_{0}\right)=0 ; \\
& \mathscr{M}\left(T_{0}, T_{1}, \ldots\right) \vDash \mathscr{H}\left(\mathscr{T}_{0}, \overline{1}\right) \leftrightarrow p\left(\mathscr{G} \mid \mathscr{T}_{0}=T_{0}\right)=1 .
\end{aligned}
$$

Then the desired $\mathscr{F} *$ is $\mathscr{H}\left(\mathscr{T}_{0}, \overline{1}\right)$.

Note that the above argument merely establishes the existence of $\mathscr{F} *$ and not its $\mathscr{M}$-definability. The $\mathscr{M}$-definability of $\mathscr{F} *$ is a consequence of the $\mathscr{M}$-definability of the probability function $p$ (restricted to sentences of $\mathscr{L}\left(\mathscr{T}_{0}, \mathscr{T}_{1}, \ldots\right)$ having at most $n$ unranked quantifiers) and the following characterization of $\mathscr{F}^{*}$ up to a set of measure $0: p\left(\mathscr{F} \leftrightarrow \mathscr{F}^{*}\right)=1 ; \mathscr{F}^{*}$ has countable ordinal rank and contains no $\mathscr{T}^{-}$ symbol not occurring in $\mathscr{F}$.

LEMMA 4.17.3 (CF. 4.7). With probability 1: the power set axiom holds in $\mathscr{M}\left(T_{0}, T_{1}, \ldots\right)$. 
Proof. Same as 4.7 save that 4.5 is replaced by 4.17 .2 .

Feferman [3] showed that if $T_{0}, T_{1}, T_{2}, \ldots$ is a sequence generic in the sense of Cohen (i.e., generic in the sense of forcing with finite conditions), then in $\mathscr{M}\left(T_{0}, T_{1}, T_{2}, \ldots\right)$, the Boolean algebra of all subsets of $\omega$ has no nonprincipal, maximal ideal. The proof of 4.18 is a measure-theoretic analogue of his argument.

THEOREM 4.18. With probability 1 : The Boolean algebra of all subsets of $\omega$ has no nonprincipal, maximal ideal in the structure $\mathscr{M}\left(T_{0}, T_{1}, T_{2}, \ldots\right)$.

Proof. Let $c \in \mathscr{C}$ be a constant which denotes a set of subsets of $\omega$. Suppose $p\left(c\right.$ is a nonprincipal, maximal ideal of $\left.2^{\omega}\right)>0$. Let $\mathscr{T}_{0}, \ldots, \mathscr{T}_{n-1}$ include all the $\mathscr{T}_{i}$-symbols occurring in $c$. By 4.17 and Fubini's theorem, there must exist sets $A_{0}, \ldots, A_{n-1}$ such that

$p\left(c\right.$ is a nonprincipal, maximal ideal of $\left.2^{\omega} \mid \mathscr{T}_{i}=A_{i}, i<n\right)=1$.

Let $d^{\prime}$ denote $\omega-d$. We intend to show

$$
p\left(\mathscr{T}_{n} \in c \& \mathscr{T}_{n}^{\prime} \in c \mid \mathscr{T}_{i}=A_{\mathfrak{i}}, i<n\right)=1 .
$$

Either $p\left(\mathscr{T}_{n} \in c \mid \mathscr{T}_{i}=A_{i}, i<n\right)$ or $p\left(\mathscr{T}_{n}^{\prime} \in c \mid \mathscr{T}_{i}=A_{i}, i<n\right)$ must be positive. Suppose the former is positive; we claim it must be equal 1 . To see the claim, let $B$ be the set of all $A$ such that

$$
p\left(\mathscr{T}_{n} \in c \mid \mathscr{T}_{i}=A_{i}, i<n, \mathscr{T}_{n}=A\right)=1 .
$$

By 4.17 and Fubini's theorem, $p\left(\mathscr{T}_{n} \in c \mid \mathscr{T}_{i}=A_{i}, i<n\right)$ equals the measure of $B \subseteq 2^{N}$. Let fin $(B)$ be the collection of all subsets of $\omega$ that differ finitely from some member of $B$. If fin $(B)=B$, then the measure of $B$ must be 0 or 1 by a standard zero-one law argument [10]. Let $f(A)$ be a subset of $\omega$ differing finitely from $A$, and let $f\left(\mathscr{T}_{n}\right)$ be a term that differs from $\mathscr{T}_{n}$ in the same way that $f(A)$ differs from $A$. By 4.16,

$$
\begin{aligned}
p\left(f\left(\mathscr{T}_{n}\right) \in c \mid\right. & \left.\mathscr{T}_{i}=A_{i}, i<n, \mathscr{T}_{n}=A\right) \\
& =p\left(\mathscr{T}_{n} \in c \mid \mathscr{T}_{i}=A_{i}, i<n, \mathscr{T}_{n}=f(A)\right) .
\end{aligned}
$$

But

$$
p\left(\mathscr{T}_{n} \in c \leftrightarrow f\left(\mathscr{T}_{n}\right) \in c \mid \mathscr{T}_{i}=A_{i}, i<n\right)=1,
$$

since $c$ denotes a nonprincipal maximal ideal with probability 1 when $\mathscr{T}_{i}$ is fixed at $A_{i}$ for all $i<n$. That establishes the claim that $p\left(\mathscr{T}_{n} \in c \mid \mathscr{T}_{i}=A_{i}, i<n\right)=1$. Another application of 4.16 gives

$$
p\left(\mathscr{T}_{n}^{\prime} \in c \mid \mathscr{T}_{i}=A_{i}, i<n\right)=1 .
$$

In order to develop Solovay's relative consistency result on the extendability of Lebesgue measure, we shall replace $\mathscr{C}$ by $\mathscr{C} *, \mathscr{L}\left(\mathscr{T}_{0}, \mathscr{T}_{1}, \mathscr{T}_{2}, \ldots\right)$ by $\mathscr{L}^{*}\left(\mathscr{T}_{0}, \mathscr{T}_{1}\right.$, $\left.\mathscr{T}_{2}, \ldots\right)$, and $\mathscr{M}\left(T_{0}, T_{1}, T_{2}, \ldots\right)$ by $\mathscr{M}^{*}\left(T_{0}, T_{1}, T_{2}, \ldots\right)$. These changes will be small but important; our sole purpose is to insure that $\mathscr{C}^{*}(1)$ is as symmetrical as 
possible from a measure-theoretic point of view. This device was originated by R. Solovay.

Let $c_{0}=c_{0}\left(\mathscr{T}_{0}, \ldots, \mathscr{T}_{n}\right)$ be an $\omega$-term of $\mathscr{C}$, that is to say, a constant of $\mathscr{C}$ which denotes a subset of $\omega$. (We can assume that the $\mathscr{T}_{i}$-symbols occurring in $c_{0}$ are $\mathscr{T}_{0}, \mathscr{T}_{1}, \ldots, \mathscr{T}_{n}$ for all sufficiently large $n$, since we can always add $\mathscr{T}_{i}$-symbols to $c_{0}$ in a spurious fashion.) Recall the notion of "sequence of independent, uniformly distributed $\omega$-terms" introduced at the end of the proof of 4.16. We say $c_{0}=$ $c_{0}\left(\mathscr{T}_{0}, \ldots, \mathscr{T}_{n}\right)$ is a projective, uniformly distributed $\omega$-term if there exist $\omega$-terms $c_{i}\left(\mathscr{T}_{0}, \ldots, \mathscr{T}_{n}\right)(1 \leqq i \leqq n)$ and $k_{i}\left(\mathscr{T}_{0}, \ldots, \mathscr{T}_{n}\right)(i \leqq n)$ of $\mathscr{C}$ such that $\left\{c_{i} \mid i \leqq n\right\}$ and $\left\{k_{i} \mid i \leqq n\right\}$ are sequences of independent, uniformly distributed $\omega$-terms and such that

$$
\mathscr{T}_{i}=k_{i}\left(c_{0}, \ldots, c_{n}\right)=c_{i}\left(k_{0}, \ldots, k_{n}\right)
$$

for all $i \leqq n .\left(k_{i}\left(c_{0}, \ldots, c_{n}\right)\right.$ is the result of substituting $c_{i}$ for $\mathscr{T}_{i}$ in $k_{i}$.) Note that if $c_{0}$ is a projective, uniformly distributed $\omega$-term by virtue of the existence of $c_{i}(1 \leqq i \leqq n)$ and $k_{i}(i \leqq n)$, then $c_{i}(1 \leqq i \leqq n)$ and $k_{i}(i \leqq n)$ also are projective, uniformly distributed $\omega$-terms. It is tedious but routine to check that $\left(\mathscr{T}_{m}\right)_{i},\left(\mathscr{T}_{m}\right)^{n}$ and $d_{n}^{m}$, which were defined immediately preceding the proof of Lemma 4.15 , are projective, uniformly distributed $\omega$-terms. The collection of all such terms is $\mathscr{M}$-definable, since $p(\mathscr{F})$, restricted to the ranked sentences of $\mathscr{L}\left(\mathscr{T}_{0}, \mathscr{T}_{1}, \mathscr{T}_{2}, \ldots\right)$, is $\mathscr{M}$-definable.

Let $\mathscr{C}^{*}(0)=\mathscr{C}(0)=\{\bar{n} \mid n \in \omega\}$. Let $\mathscr{C}^{*}(1)$ be the set of all projective, uniformly distributed $\omega$-terms. In the previous paragraph it was remarked that $\mathscr{C}^{*}(1)$ is $\mathscr{M}$-definable. It is convenient to make $\mathscr{C}^{*}(1)$ a set of $\mathscr{M}$ by the following device. The proof for the power set axiom for $\mathscr{M}\left(T_{0}, T_{1}, T_{2}, \ldots\right)$ (essentially the argument of 4.7) shows that there exists a set $b \in \mathscr{M}$ of $\omega$-terms such that with probability 1 , each $\omega$-term of $\mathscr{C}$ is equal to some $\omega$-term of $b$. Thus there is no harm in restricting the concept of $\omega$-term to $b . \mathscr{C}^{*}(\gamma+1)(\gamma>0)$ and $\mathscr{C}^{*}(\lambda)(\lambda$ a limit ordinal $)$ are defined in the same manner as $\mathscr{C}(\gamma+1)$ and $\mathscr{C}(\lambda)$. The constants of $\mathscr{C}^{*}(\gamma+1)(\gamma>0)$ denote the first-order definable subsets of $\mathscr{M}_{\gamma+1}^{*}\left(T_{0}, T_{1}, T_{2}, \ldots\right)$. The atomic formulas of $\mathscr{L}^{*}\left(\mathscr{T}_{0}, \mathscr{T}_{1}, \mathscr{T}_{2}, \ldots\right)$ are of the form $t_{1} \in t_{2}$, where $t_{1}$ and $t_{2}$ are variables or constants of $\mathscr{C}^{*}$.

Let $\mathscr{M}_{0}^{*}\left(T_{0}, T_{1}, T_{2}, \ldots\right)=\omega$. Let $t \in \mathscr{C}^{*}(1)$; suppose $\left\{\mathscr{T}_{i} \mid i \in K\right\}$ is the set of all $\mathscr{T}_{i}$-symbols occurring in $t$. By Lemma 4.17 ,

$$
p\left(\bar{n} \in t \mid \mathscr{T}_{i}=T_{i}, i \in K\right)=0 \text { or } 1 .
$$

We say that $t$ denotes $\left\{n \mid p\left(\bar{n} \in t \mid \mathscr{T}_{i}=T_{i}, i \in K\right)=1\right\} . \mathscr{M}_{1}^{*}\left(T_{0}, T_{1}, T_{2}, \ldots\right)$ is the set of all sets denoted by some $t \in \mathscr{C}^{*}(1) . \mathscr{M}_{\gamma+1}^{*}\left(T_{0}, T_{1}, T_{2}, \ldots\right)(\gamma>0)$ is defined in the same inductive fashion as $\mathscr{M}_{\gamma+1}\left(T_{0}, T_{1}, T_{2}, \ldots\right)$. Thus the only conceptual difference between $\mathscr{M}\left(T_{0}, T_{1}, T_{2}, \ldots\right)$ and $\mathscr{M}^{*}\left(T_{0}, T_{1}, T_{2}, \ldots\right)$, or between $\mathscr{L}\left(\mathscr{T}_{0}, \mathscr{T}_{1}\right.$, $\left.\mathscr{T}_{2}, \ldots\right)$ and $\mathscr{L}^{*}\left(\mathscr{T}_{0}, \mathscr{T}_{1}, \mathscr{T}_{2}, \ldots\right)$, is the difference between $\mathscr{C}(1)$ and $\mathscr{C}^{*}(1)$.

A routine transfinite induction establishes the existence of an $\mathscr{M}$-definable $f: \mathscr{C}^{*} \rightarrow \mathscr{C}$ with the following properties for each $c \in \mathscr{C}^{*}:$ if $c$ is an $\omega$-term, then $f(c)$ 
is an $\omega$-term; $c$ and $f(c)$ have the same occurrences of $\mathscr{T}_{j}$-symbols; the set denoted by $c$ in $\mathscr{M}^{*}\left(T_{0}, T_{1}, \ldots\right)$ equals the set denoted by $f(c)$ in $\mathscr{M}\left(T_{0}, T_{1}, \ldots\right)$ for all $\left(T_{0}, T_{1}, \ldots\right)$.

For each sentence $\mathscr{F}$ of $\mathscr{L}^{*}\left(\mathscr{T}_{0}, \mathscr{T}_{1}, \mathscr{T}_{2}, \ldots\right)$, the probability that $\mathscr{F}$ is true in $\mathscr{M}^{*}\left(T_{0}, T_{1}, T_{2}, \ldots\right)$, denoted by $p^{*}(\mathscr{F})$, is the measure of the Borel set

$$
\left\{\left(T_{0}, T_{1}, T_{2}, \ldots\right) \mid \mathscr{M}^{*}\left(T_{0}, T_{1}, T_{2}, \ldots\right) \vDash \mathscr{F}\right\} .
$$

The argument of 4.1 shows: for each $n \geqq 0$, the function $p^{*}(\mathscr{F})$, restricted to sentences of $\mathscr{L}^{*}\left(\mathscr{T}_{0}, \mathscr{T}_{1}, \mathscr{T}_{2}, \ldots\right)$ having at most $n$ unranked quantifiers, is $\mathscr{M}$ definable. Note that the peculiar definition of $\mathscr{C}^{*}(1)$ requires the use of the $\mathscr{M}$ definability of $p$ for ranked sentences of $\mathscr{L}\left(\mathscr{T}_{0}, \mathscr{T}_{1}, \mathscr{T}_{2}, \ldots\right)$ in the proof of the $\mathscr{M}$-definability of $p^{*}$. The arguments of 4.17.1-3 show that with probability 1 , $\mathscr{M}^{*}\left(T_{0}, T_{1}, T_{2}, \ldots\right)$ is a model of ZF. Observe that if $t \in \mathscr{C}^{*}(1) \subseteq \mathscr{C}$, then $p^{*}(\bar{n} \in t)$ $=p(\bar{n} \in t)$ for all $n \in \omega$.

LEMMA 4.19. Let $r$ be a map of $\mathscr{C}^{*}(1)$ onto $\mathscr{C}^{*}(1)$ such that $r^{*}:\left(2^{N}\right)^{N} \rightarrow\left(2^{N}\right)^{N}$ is measure-preserving. Suppose that fixing $\left\{\mathscr{T}_{i} \mid i \in K\right\}$ has the effect of fixing

$$
\left\{r\left(\mathscr{T}_{i}\right) \mid i \in K_{r}\right\}
$$

Then for each sentence $\mathscr{F}$ of $\mathscr{L}^{*}\left(\mathscr{T}_{0}, \mathscr{T}_{1}, \mathscr{T}_{2}, \ldots\right)$,

$$
p\left(r(\mathscr{F}) \mid \mathscr{T}_{i}=A_{i}, i \in K\right)=p\left(\mathscr{F} \mid \mathscr{T}_{i}=B_{i}, i \in K_{r}\right),
$$

where for each $i \in K_{r}, B_{i}$ is the set denoted by $r\left(\mathscr{T}_{i}\right)$ in $\mathscr{M}^{*}\left(T_{0}, T_{1}, T_{2}, \ldots\right)$ when $T_{i}=A_{i}$ for all $i \in K$.

Proof. Same as 4.16. The arguments of 4.11 and 4.12 are correct for $\mathscr{M}^{*}\left(T_{0}, T_{1}, T_{2}, \ldots\right)$. The differences between $\mathscr{C}(1)$ and $\mathscr{C}^{*}(1)$ are irrelevant.

LEMMA 4.20. Let $\left\{\mathscr{T}_{i} \mid i \in K\right\}$ include all the $\mathscr{T}_{i}$-symbols occurring in the sentence $\mathscr{F}$ of $\mathscr{L}^{*}\left(\mathscr{T}_{0}, \mathscr{T}_{1}, \mathscr{T}_{2}, \ldots\right)$. Then for every sequence $\left\{A_{\mathfrak{i}} \mid i \in K\right\}$ of subsets of $\omega$; $p\left(\mathscr{F} \mid \mathscr{T}_{i}=A_{i}, i \in K\right)=0$ or 1 .

Proof. Same as 4.17. But it is necessary to check that the maps of $\mathscr{C}(1)$ onto $\mathscr{C}(1)$ employed in the proof of 4.17 are also maps of $\mathscr{C}^{*}(1)$ onto $\mathscr{C}^{*}(1)$. This is a routine matter, since all the relevant terms of $\mathscr{C}\left(\omega-\mathscr{T}_{1}\right.$, etc.) are projective, uniformly distributed $\omega$-terms.

LemMa 4.21. With probability 1 , the dependent axiom of choice holds in $\mathscr{M}^{*}\left(T_{0}\right.$, $\left.T_{1}, T_{2}, \ldots\right)$.

Proof. Same as 4.15. But it is necessary to check that the maps of $\mathscr{C}(1)$ onto $\mathscr{C}(1)$ employed in the proofs of 4.14 and 4.15 are also maps of $\mathscr{C}^{*}(1)$ onto $\mathscr{C}^{*}(1)$.

From now on we will identify elements of $2^{N}$ with real numbers in the closed unit interval $[0,1]$ by means of the usual dyadic expansions. Thus if $A, B \in 2^{N}$, then $A+B(\bmod 1)$ will correspond to the arithmetic sum (mod 1$)$ of the real numbers corresponding to $A$ and $B$. A similar remark applies to $\omega$-terms of $\mathscr{C}^{*}$. 
Lemma 4.22. (a) Let $c\left(\mathscr{T}_{0}, \ldots, \mathscr{T}_{n}\right) \in \mathscr{C}^{*}(1)$; then there exists a map $r$ of $\mathscr{C}^{*}(1)$ onto $\mathscr{C}^{*}(1)$ such that $r\left(\mathscr{T}_{0}\right)=c\left(\mathscr{T}_{0}, \ldots, \mathscr{T}_{n}\right), r\left(\mathscr{T}_{1}\right)=\mathscr{T}_{n+1}$, and the induced map $r^{*}:\left(2^{N}\right)^{N} \rightarrow\left(2^{N}\right)^{N}$ is measure-preserving.

(b) If $t\left(\mathscr{T}_{0}, \ldots, \mathscr{T}_{n-1}\right)$ is an $\omega$-term of $\mathscr{C}^{*}$, then there exists a $c_{n} \in \mathscr{C}^{*}(1)$ such that $c_{n}=\mathscr{T}_{n}+t\left(\mathscr{T}_{0}, \ldots, \mathscr{T}_{n-1}\right)(\bmod 1)$ with probability 1. In addition, there exists a map $r$ of $\mathscr{C}^{*}(1)$ onto $\mathscr{C}^{*}(1)$ such that $r\left(\mathscr{T}_{i}\right)=\mathscr{T}_{i}(i<n), r\left(\mathscr{T}_{n}\right)=c_{n}$, and the induced map $r^{*}:\left(2^{N}\right)^{N} \rightarrow\left(2^{N}\right)^{N}$ is measure-preserving.

Proof. Since $c_{0}=c\left(\mathscr{T}_{0}, \ldots, \mathscr{T}_{n}\right) \in \mathscr{C}^{*}(1)$, there must exist $c_{i}\left(\mathscr{T}_{0}, \ldots, \mathscr{T}_{n}\right)$ and $k_{i}\left(\mathscr{T}_{0}, \ldots, \mathscr{T}_{n}\right)(i \leqq n)$ such that $\left\{c_{i} \mid i \leqq n\right\}$ and $\left\{k_{i} \mid i \leqq n\right\}$ are sequences of independent, uniformly distributed $\omega$-terms of $\mathscr{C}^{*}(1)$ and such that $\mathscr{T}_{i}=k_{i}\left(c_{0}, \ldots, c_{n}\right)$ $(i \leqq n)$. Then the map $r$, defined by

$$
\begin{aligned}
r\left(\mathscr{T}_{0}\right) & =c\left(\mathscr{T}_{0}, \ldots, \mathscr{T}_{n}\right)=c_{0}, \\
r\left(\mathscr{T}_{1}\right) & =\mathscr{T}_{n+1}, r\left(\mathscr{T}_{n+k}\right)=\mathscr{T}_{n+k} \quad(k>1), \\
r\left(\mathscr{T}_{i+1}\right) & =c_{i} \quad(1 \leqq i \leqq n),
\end{aligned}
$$

maps $\mathscr{C}^{*}(1)$ onto $\mathscr{C}^{*}(1)$. The induced map $r^{*}:\left(2^{N}\right)^{N} \rightarrow\left(2^{N}\right)^{N}$ is measure-preserving, since $\left\{r\left(\mathscr{T}_{i}\right) \mid i \in \omega\right\}$ is a sequence of independent, uniformly distributed $\omega$-terms. (Cf. end of proof of 4.16.)

By the remarks immediately preceding 4.19 , there exists an $\omega$-term $t_{1}\left(\mathscr{T}_{0}, \ldots\right.$, $\left.\mathscr{T}_{n-1}\right) \in \mathscr{C}$ such that $\mathscr{T}_{n}+t\left(\mathscr{T}_{0}, \ldots, \mathscr{T}_{n-1}\right)$ denotes the same set in $\mathscr{M}^{*}\left(T_{0}, T_{1}, \ldots\right)$ that $\mathscr{T}_{n}+t_{1}\left(\mathscr{T}_{0}, \ldots, \mathscr{T}_{n-1}\right)$ denotes in $\mathscr{M}\left(T_{0}, T_{1}, \ldots\right)$. We must show $c_{n}=\mathscr{T}_{n}+t_{1}$ is a projective, uniformly distributed $\omega$-term. For this purpose, it is convenient to think of the $\omega$-terms of $\mathscr{C}$ as random variables [5] defined on the sample space $\left(2^{N}\right)^{N}$. An $\omega$-term is uniformly distributed in our sense if and only if it is uniformly distributed in the standard sense when it is interpreted as a random variable. Thus if $c_{0}, \ldots, c_{n}$ is a sequence of uniformly distributed $\omega$-terms such that $c_{i}$ and $c_{j}$ $(0 \leqq i<j \leqq n)$ have no $\mathscr{T}_{k}$-symbols in common, then $c_{0}, \ldots, c_{n}$ is a sequence of independent, uniformly distributed $\omega$-terms (cf. Halmos [10, p. 194]). We claim

$$
\mathscr{T}_{0}, \ldots, \mathscr{T}_{n-1}, \mathscr{T}_{n}+t_{1}\left(\mathscr{T}_{0}, \ldots, \mathscr{T}_{n-1}\right), \mathscr{T}_{n+1}, \ldots
$$

is a sequence of independent, uniformly distributed $\omega$-terms. The claim is a consequence of the following type of standard argument. Let $X_{0}, X_{1}, Y$ be random variables on $\left(2^{N}\right)^{N}$ such that $X_{0}, X_{1}$ are independent and uniformly distributed, and such that $X_{0}, Y$ are independent. Then $X_{0}+Y(\bmod 1), X_{1}$ are independent and uniformly distributed, since

$$
\begin{aligned}
p\left(X_{0}+Y=a \& X_{1}=c\right) & =\sum_{b} p\left(Y=a-b \& X_{0}=b \& X_{1}=c\right) \\
& =p\left(X_{0}=a\right) p\left(X_{1}=c\right) \sum_{b} p\left(Y=a-b \mid X_{0}=b \& X_{1}=c\right) \\
& =p\left(X_{0}=a\right) p\left(X_{1}=c\right) .
\end{aligned}
$$

To check that $c_{n}=\mathscr{T}_{n}+t_{1}\left(\mathscr{T}_{0}, \ldots, \mathscr{T}_{n-1}\right)$ is projective and uniformly distributed, 
let $c_{i}=k_{i}=\mathscr{T}_{i}(i<n)$ and let $k_{n}=\mathscr{T}_{n}-t_{1}\left(\mathscr{T}_{0}, \ldots, \mathscr{T}_{n-1}\right)(\bmod 1)$. The desired map $r$ is defined by $r\left(\mathscr{T}_{i}\right)=\mathscr{T}_{i}(i \neq n)$ and $r\left(\mathscr{T}_{n}\right)=c_{n}$.

Let $x^{\Omega}$ be a restricted variable of $\mathscr{L}^{*}\left(\mathscr{T}_{0}, \mathscr{T}_{1}, \ldots\right)$, restricted to the subsets of $\omega$ in $\mathscr{M}^{*}\left(T_{0}, T_{1}, \ldots\right)$. Thus we can denote each subset of $2^{N}$ in $\mathscr{M}^{*}\left(T_{0}, T_{1}, \ldots\right)$ by a constant of the form $\hat{x}^{\Omega \mathscr{F}}\left(x^{\Omega}\right)$, where $\mathscr{F}\left(x^{\Omega}\right)$ is a formula of $\mathscr{L}^{*}\left(\mathscr{T}_{0}, \mathscr{T}_{1}, \ldots\right)$ whose only free variable is $x^{\Omega}$. For each $(T)=\left(T_{0}, T_{1}, T_{2}, \ldots\right)$, we define the absolute (in $T$ ) measure of $\hat{x}^{\Omega} \mathscr{F}\left(x^{\Omega}\right)$, denoted by $\mu_{a}^{T}\left(\hat{x}^{\Omega} \mathscr{F}\left(x^{\Omega}\right)\right)$, as follows:

$$
\mu_{a}^{T}\left(\hat{x}^{\Omega \mathscr{F}}\left(x^{\Omega}\right)\right)=p^{*}\left(\mathscr{F}\left(\mathscr{T}_{j}\right) \mid \mathscr{T}_{i}=T_{i}, i \in K\right),
$$

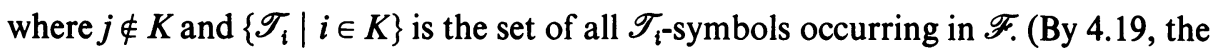
value of $j$ is irrelevant so long as $j \notin K$.) For each $(T)=\left(T_{0}, T_{1}, T_{2}, \ldots\right)$, we say $\mathscr{M}^{*}\left(T_{0}, T_{1}, T_{2}, \ldots\right)$ is measure-complete if for every $\mathscr{F}\left(x^{\Omega}\right) \in \mathscr{L}^{*}\left(\mathscr{T}_{0}, \mathscr{T}_{1}, \ldots\right)$, we have

$$
\mu_{a}^{T}\left(\hat{x}^{\Omega} \mathscr{F}\left(x^{\Omega}\right)\right)>0 \rightarrow \mathscr{M}^{*}\left(T_{0}, T_{1}, \ldots\right) \vDash\left(E x^{\Omega}\right) \mathscr{F}\left(x^{\Omega}\right) .
$$

The concept of absolute (in $T$ ) measure has been defined for definitions of subsets of $2^{N}$ in $\mathscr{M}^{*}\left(T_{0}, T_{1}, \ldots\right)$ rather than subsets of $2^{N}$; for each $(T)=\left(T_{0}, T_{1}, \ldots\right)$, let us say $\mu_{a}^{T}$ is well-defined on all subsets of $2^{N}$ if all definitions of any given subset of $2^{N}$ in $\mathscr{M}^{*}\left(T_{0}, T_{1}, \ldots\right)$ have the same absolute (in $\left.T\right)$ measure.

LEMMA 4.23. With probability 1: (a) $\mathscr{M}^{*}\left(T_{0}, T_{1}, T_{2}, \ldots\right)$ is measure-complete; (b) if $\mathscr{M}^{*}\left(T_{0}, T_{1}, T_{2}, \ldots\right)$ is measure-complete, then $\mu_{a}^{T}$ is well-defined on all subsets of $2^{N}$ in $\mathscr{M}^{*}\left(T_{0}, T_{1}, T_{2}, \ldots\right)$.

Proof. First we do (a). Let $\mathscr{T}_{0}$ be the only $\mathscr{T}_{i}$-symbol occurring in $\mathscr{F}\left(x^{\Omega}, \mathscr{T}_{0}\right)$. We must show: for almost all $(T)=\left(T_{0}, T_{1}, T_{2}, \ldots\right)$, if $\mu_{a}^{T}\left(\hat{x}^{\Omega} \mathscr{F}\left(x^{\Omega}, \mathscr{T}_{0}\right)\right)>0$, then $\mathscr{M}^{*}\left(T_{0}, T_{1}, T_{2}, \ldots\right) \vDash\left(E x^{\Omega}\right) \mathscr{F}\left(x^{\Omega}, \mathscr{T}_{0}\right)$. Suppose not. Then by 4.20 and Fubini's theorem, there exists a set $K \subseteq 2^{N}$ of positive measure such that for all $T_{0} \in K$,

$$
\begin{aligned}
p^{*}\left(\sim\left(E x^{\Omega}\right) \mathscr{F}\left(x^{\Omega}, \mathscr{T}_{0}\right) \mid \mathscr{T}_{0}\right. & \left.=T_{0}\right)=1, \\
p^{*}\left(\mathscr{F}\left(\mathscr{T}_{1}, \mathscr{T}_{0}\right) \mid \mathscr{T}_{0}=T_{0}\right) & >0 .
\end{aligned}
$$

Now we do (b). Let $\mathscr{F}_{0}\left(x^{\Omega}, \mathscr{T}_{0}\right)$ and $\mathscr{F}_{1}\left(x^{\Omega}, \mathscr{T}_{1}\right)$ be formulas such that $\mathscr{T}_{i}$ is the only $\mathscr{T}_{i}$-symbol occurring in $\mathscr{F}_{i}\left(x^{\Omega}, \mathscr{T}_{i}\right)(i<2)$. By 4.20 and Fubini's theorem,

$$
m_{0}=\mu_{a}^{T}\left(\hat{x}^{\Omega} \mathscr{F}_{0}\left(x^{\Omega}, \mathscr{T}_{0}\right)\right)=p^{*}\left(\mathscr{F}_{0}\left(\mathscr{T}_{2}, \mathscr{T}_{0}\right) \mid \mathscr{T}_{0}=T_{0}, \mathscr{T}_{1}=T_{1}\right)
$$

for almost all $(T)=\left(T_{0}, T_{1}, T_{2}, \ldots\right)$. Similarly,

$$
m_{1}=\mu_{a}^{T}\left(\hat{x}^{\Omega} \mathscr{F}_{1}\left(x^{\Omega}, \mathscr{T}_{1}\right)\right)=p^{*}\left(\mathscr{F}_{1}\left(\mathscr{T}_{2}, \mathscr{T}_{1}\right) \mid \mathscr{T}_{0}=T_{0}, \mathscr{T}_{1}=T_{1}\right)
$$

for almost all $(T)$. Let $\mathscr{F}_{0} \triangle \mathscr{F}_{1}$ denote $\left(\mathscr{F}_{0} \& \sim \mathscr{F}_{1}\right) \vee\left(\mathscr{F}_{1} \& \sim \mathscr{F}_{0}\right)$. Then for almost all $(T)$, if

$$
m_{\triangle}=\mu_{a}^{T}\left(\hat{x}^{\Omega}\left(\mathscr{F}_{0}\left(x^{\Omega}, \mathscr{T}_{0}\right) \triangle \mathscr{F}_{1}\left(x^{\Omega}, \mathscr{T}_{1}\right)\right)\right)=0,
$$


then $m_{0}=m_{1}$. Finally, for all $(T)=\left(T_{0}, T_{1}, T_{2}, \ldots\right)$, if $\mathscr{M}^{*}\left(T_{0}, T_{1}, T_{2}, \ldots\right)$ is measure-complete and

$$
\mathscr{M}^{*}\left(T_{0}, T_{1}, T_{2}, \ldots\right) \vDash\left(x^{\Omega}\right)\left(\mathscr{F}_{0}\left(x^{\Omega}, \mathscr{T}_{0}\right)=\mathscr{F}_{1}\left(x^{\Omega}, \mathscr{T}_{1}\right)\right),
$$

then $m_{\triangle}=0$.

Lemma 4.23 strongly suggests that $\mu_{a}^{T}$ is a good candidate for a countably additive, translation-invariant extension of Lebesgue measure to all subsets of $2^{N}$ in $\mathscr{M}^{*}\left(T_{0}, T_{1}, T_{2}, \ldots\right)$ for almost all $(T)=\left(T_{0}, T_{1}, T_{2}, \ldots\right)$. We shall of course carry out this suggestion, but there is a delicate problem, easily overlooked, that we must face immediately. The definition of $\mu_{a}^{T}$ was given by means of terms denoting subsets of $2^{N}$ in $\mathscr{M}^{*}\left(T_{0}, T_{1}, T_{2}, \ldots\right)$. With probability 1 , there is no map in $\mathscr{M}^{*}\left(T_{0}, T_{1}, T_{2}, \ldots\right)$ which associates with each subset of $2^{N}$ a term denoting that subset; this follows from the argument of Theorem 4.18. Nonetheless, we are able to define $\mu_{a}^{T}$ in $\mathscr{M}\left(T_{0}, T_{1}, \ldots\right)$ for almost all $(T)=\left(T_{0}, T_{1}, \ldots\right)$ by exploiting the measure-theoretic symmetries of $\mathscr{C}^{*}(1)$. In fact, Lemma 4.24 is the sole reason we insisted that every $\omega$-term of $\mathscr{C}^{*}(1)$ be projective and uniformly distributed.

LeMmA 4.24. With probability $1: \mu_{a}^{T}$ is $\mathscr{M}\left(T_{0}, T_{1}, \ldots\right)$-definable.

Proof. Let $s$ be an arbitrary subset of $2^{N}$ in $\mathscr{M}^{*}\left(T_{0}, T_{1}, T_{2}, \ldots\right)$. For almost all $(T)=\left(T_{0}, T_{1}, T_{2}, \ldots\right)$, there exist $\mathscr{M}^{*}\left(T_{0}, T_{1}, T_{2}, \ldots\right)$-definable maps $f$ and $b$ such that $f(s)=R\left(x^{\Omega}, y\right)$ is a ranked formula of $\mathscr{L}^{*}\left(\mathscr{T}_{0}, \mathscr{T}_{1}, \ldots\right)$ in which no $\mathscr{T}_{i}$-symbols occur, $b(s)=b$ is a subset of $\omega$ in $\mathscr{M}^{*}\left(T_{0}, T_{1}, T_{2}, \ldots\right)$, and $\hat{x}^{\Omega} R\left(x^{\Omega}, y\right)$ denotes $s$ in $\mathscr{M}^{*}\left(T_{0}, T_{1}, T_{2}, \ldots\right)$ when $y$ is interpreted as $b$. The set $b$ is merely an encoding of the finitely many $T_{i}$ 's that are denoted by $\mathscr{T}_{i}$-symbols in some term denoting $s$; it is easily checked that all such encodings can be accomplished by projective, uniformly distributed $\omega$-terms (cf. $d_{n}^{m}$ defined immediately preceding 4.15). Thus $b$ is denoted by some term $c\left(\mathscr{T}_{0}, \mathscr{T}_{1}, \mathscr{T}_{2}\right) \in \mathscr{C}^{*}(1)$, and

$$
\mu_{a}^{T}(s)=p^{*}\left(R\left(\mathscr{T}_{3}, c\left(\mathscr{T}_{0}, \mathscr{T}_{1}, \mathscr{T}_{2}\right)\right) \mid \mathscr{T}_{i}=T_{i}, i \leqq 2\right) .
$$

Unfortunately there is (with probability 1) no $\mathscr{M}^{*}\left(T_{0}, T_{1}, \ldots\right)$-definable map $h$ such that $h(r)=c\left(\mathscr{T}_{0}, \mathscr{T}_{1}, \mathscr{T}_{2}\right)$. But, thanks to the symmetry of $\mathscr{C}^{*}(1)$, we don't need $c$. We claim:

$$
\mu_{a}^{T}(s)=p^{*}\left(R\left(\mathscr{T}_{1}, \mathscr{T}_{0}\right) \mid \mathscr{T}_{0}=b\right) .
$$

The claim follows from 4.22 (a), 4.19, and Fubini's theorem. Finally, note that the conditional probability function $p^{*}\left(\mathscr{F} \mid \mathscr{T}_{0}=b\right)$, restricted to ranked sentences $\mathscr{F} \in \mathscr{L}^{*}\left(\mathscr{T}_{0}, \mathscr{T}_{1}, \ldots\right)$ and sets $b \in \mathscr{M}^{*}\left(T_{0}, T_{1}, \ldots\right)$, is $\mathscr{M}^{*}\left(T_{0}, T_{1}, \ldots\right)$-definable for almost all $\left(T_{0}, T_{1}, \ldots\right)$ by a routine modification of the argument of 4.1 .

LEMMA 4.25. With probability 1: $\mu_{a}^{T}$ is countably additive, translation-invariant, and agrees with Lebesgue measure on all Lebesgue-measurable sets.

Proof. Let $\mathscr{F}\left(x^{\Omega}, \mathscr{T}_{0}, x^{0}\right)$ be a formula of $\mathscr{L}^{*}\left(\mathscr{T}_{0}, \mathscr{T}_{1}, \ldots\right)$ whose only $\mathscr{T}_{i}$-symbol is $\mathscr{T}_{0}$. Let $A \subseteq \omega$ be such that for all $m \neq n$,

$$
p^{*}\left(\left(x^{\Omega}\right) \sim\left[\mathscr{F}\left(x^{\Omega}, \mathscr{T}_{0}, \bar{m}\right) \& \mathscr{F}\left(x^{\Omega}, \mathscr{T}_{0}, \bar{n}\right)\right] \mid \mathscr{T}_{0}=A\right)=1 .
$$


Let $(T)=\left(A, T_{1}, T_{2}, \ldots\right)$. Then

$$
\begin{aligned}
\sum_{n} \mu_{a}^{T}\left(\hat{x}^{\Omega} \mathscr{F}\left(x^{\Omega}, \mathscr{T}_{0}, \bar{n}\right)\right) & =\sum_{n} p^{*}\left(\mathscr{F}\left(\mathscr{T}_{1}, \mathscr{T}_{0}, \bar{n}\right) \mid \mathscr{T}_{0}=A\right) \\
& =p^{*}\left(\left(E x^{0}\right) \mathscr{F}\left(\mathscr{T}_{1}, \mathscr{T}_{0}, x^{0}\right) \mid \mathscr{T}_{0}=A\right) \\
& =\mu_{a}^{T}\left(\hat{x}^{\Omega}\left(E x^{0}\right) \mathscr{F}\left(x^{\Omega}, \mathscr{T}_{0}, x^{0}\right)\right) .
\end{aligned}
$$

The countable additivity of $\mu_{a}^{T}$ for almost all $(T)=\left(T_{0}, T_{1}, T_{2}, \ldots\right)$ now follows from 4.20 and Fubini's theorem.

We establish the translation-invariance of $\mu_{a}^{T}$ for almost all $(T)$ by a variation of the argument of 4.24 . Let $\mathscr{F}\left(x^{\Omega}, \mathscr{T}_{0}\right)$ be a formula of $\mathscr{L}^{*}\left(\mathscr{T}_{0}, \mathscr{T}_{1}, \ldots\right)$ whose only $\mathscr{T}_{i}$-symbol is $\mathscr{T}_{0}$, and let $c\left(\mathscr{T}_{0}, \mathscr{T}_{1}\right)$ be an $\omega$-constant whose only $\mathscr{T}_{i}$-symbols are $\mathscr{T}_{0}$ and $\mathscr{T}_{1}$. Then

$$
\begin{aligned}
\mu_{c} & =\mu_{a}^{T}\left(\hat{x}^{\Omega} \mathscr{F}\left(x^{\Omega}+c\left(\mathscr{T}_{0}, \mathscr{T}_{1}\right), \mathscr{T}_{0}\right)\right) \\
& =p^{*}\left(\mathscr{F}\left(\mathscr{T}_{2}+c\left(\mathscr{T}_{0}, \mathscr{T}_{1}\right), \mathscr{T}_{0}\right) \mid \mathscr{T}_{i}=T_{i}, i \leqq 1\right)
\end{aligned}
$$

for all $(T)=\left(T_{0}, T_{1}, \ldots\right)$; and

$$
\mu=\mu_{a}^{T}\left(\hat{x}^{\Omega} \mathscr{F}\left(x^{\Omega}, \mathscr{T}_{0}\right)\right)=p^{*}\left(\mathscr{F}\left(\mathscr{T}_{2}, \mathscr{T}_{0}\right) \mid \mathscr{T}_{i}=T_{i}, i \leqq 1\right)
$$

for almost all $(T)$ by 4.20 and Fubini's theorem. By 4.22 (b) and 4.19,

$$
\begin{aligned}
p^{*}\left(\mathscr{F}\left(\mathscr{T}_{2}+c\left(\mathscr{T}_{0}, \mathscr{T}_{1}\right), \mathscr{T}_{0}\right) \mid \mathscr{T}_{i}=T_{i}, i \leqq 2\right) \\
\quad=p^{*}\left(\mathscr{F}_{2}\left(\mathscr{T}_{2}, \mathscr{T}_{0}\right) \mid \mathscr{T}_{i}=T_{i}, i \leqq 1, \mathscr{T}_{2}=T_{2}+c\left(T_{0}, T_{1}\right)\right)
\end{aligned}
$$

for almost all $(T)$. But then by Fubini's theorem $\mu_{c}=\mu$ for almost all $T$.

For almost all $(T), \mu_{a}^{T}$ agrees with Lebesgue measure on all Lebesgue-measurable sets because $\mu_{a}^{T}$ is countably additive and agrees with Lebesgue measure on all basic open subsets of $2^{N}$. (Of course we are using the fact that every countably additive measure on $2^{N}$ is regular (Halmos [10]); this fact requires the countable axiom of choice in its proof; fortunately, 4.21 provides us with the countable axiom of choice for almost all $(T)$.)

THEOREM 4.26 (Solovay [29], [30]). If $Z F$ is consistent, then $Z F+$ " there exists a countably additive, translation-invariant extension of Lebesgue measure to all sets of reals" + "dependent axiom of choice" is consistent.

Proof. By 4.21, 4.24 and $4.25, \mathscr{M}^{*}\left(T_{0}, T_{1}, T_{2}, \ldots\right)$ is the desired model with probability one.

Scott and Solovay [25], [26] have devised a very valuable way of viewing forcing arguments in terms of homomorphisms of Boolean algebras. Consider what happens from their point of view when a Cohen-generic $T \subseteq N$ is added to $\mathscr{M}$. Cohen [1] defined a forcing relation, $q \vDash \mathscr{F}$, where $q$ is a finite set of conditions on $T$ and $\mathscr{F}$ is a sentence of $\mathscr{L}(\mathscr{T})$. Suppose $q$ is interpreted as a basic open subset of $2^{N}$. Then

$$
\phi(\mathscr{F})=\bigcup\{q \mid q \vDash \sim \sim \mathscr{F}\}
$$


defines a map from the sentences of $\mathscr{L}(\mathscr{T})$ into RO, the Boolean algebra of regular open subsets of $2^{N}$. RO is a complete, Boolean algebra, and $\phi$ is a homomorphism in the sense of complete Boolean algebras. By that we mean, $\phi\left(\mathscr{F}_{1} \vee \mathscr{F}_{2}\right)=\phi\left(\mathscr{F}_{1}\right)$ $\vee \phi\left(\mathscr{F}_{2}\right) . \phi(\sim \mathscr{F})=$ interior of $2^{N}-\phi(\mathscr{F})$, and $\phi\left(\left(E x^{\alpha}\right) \mathscr{F}\left(x^{\alpha}\right)\right)=\bigcup\{\phi(c) \mid c \in \mathscr{C}(\alpha)\}$. Cohen's key lemma is: for each $n \geqq 0$, the map $\phi$, restricted to sentences having at most $n$ unranked quantifiers, is $\mathscr{M}$-definable. The $\mathscr{M}$-definability of $\phi$, together with the fact that $\phi$ is a homomorphism in the sense of complete Boolean algebras, is all that is used to show $\phi(\mathscr{F})=1$ for each instance $\mathscr{F}$ of the replacement axiom.

Our probability function $p(\mathscr{F})$ is a map from the sentences of $\mathscr{L}(\mathscr{T})$ into the usual linear ordering of the reals in $[0,1]$. Now $p$ is not a homomorphism, since $p\left(\mathscr{F}_{1} \vee \mathscr{F}_{2}\right)$ need not equal

$$
\max \left(p\left(\mathscr{F}_{1}\right), p\left(\mathscr{F}_{2}\right)\right)
$$

But $p$ is a "near-homomorphism"; it preserves negations and monotonic unions: $p(\sim \mathscr{F})=1-p(\mathscr{F})$, and $p\left(\left(E x^{\alpha}\right) \mathscr{F}\left(x^{\alpha}\right)\right)=$ least upper bound of $\left\{p\left(\mathscr{F}\left(c_{0}\right) \vee \cdots\right.\right.$ $\left.\left.\vee \mathscr{F}\left(c_{n}\right)\right) \mid c_{i} \in \mathscr{C}(\alpha)\right\}$. The fact that $p$ is not a homomorphism is exactly the reason that prenex normal form was exploited so heavily in the proof (Lemma 4.1) of the $\mathscr{M}$-definability of $p$. The $\mathscr{M}$-definability of $\phi$, together with the fact that $p$ is a "near-homomorphism", was enough to show (Lemmas 4.2 and 4.3 ) that $p(\mathscr{F})=1$ for each instance $\mathscr{F}$ of the replacement axiom. Thus the measure-theoretic uniformity approach seems to suggest that the Scott-Solovay Boolean-valued model point of view can be generalized still further, possibly by dropping homomorphisms in favor of "near-homomorphisms".

Solovay [29] significantly extended Cohen's forcing method by using closed subsets of $2^{N}$ of positive measure as forcing conditions. His forcing relation, $q \Vdash \mathscr{F}$, is identical with Cohen's save that $q$ is a set of natural numbers of $\mathscr{M}$ which encodes a closed subset of $2^{N}$ of positive measure. The proof of Solovay's Theorem (4.26) is based on this notion of forcing. Call $T \subseteq N$ Solovay-generic if it is generic in his sense of forcing. Call $T$ fundamental if for all sentences $\mathscr{F}$ of $\mathrm{ZF}, p(\mathscr{F})$ $=1 \leftrightarrow \mathscr{M}(T) \vDash \mathscr{F}$. (By Corollary $4.17, p(\mathscr{F})=1$ or 0 if $\mathscr{F}$ has no $\mathscr{T}_{i}$-symbols.) Solovay has observed that $T$ is fundamental if and only if $T$ has the same degree of nonconstructibility as some Solovay-generic $T^{\prime}$. (In this case, $T$ and $T^{\prime}$ have the same degree if each is constructible from the other via the ordinals of $\mathscr{M}$.) Thus the transition described in the previous paragraph from homomorphisms to "near-homomorphisms" might correspond to a transition from sets to degrees of sets.

\section{REFERENCES}

1. J. W. Addison, The undefinability of the definable, Abstract 622-71, Notices Amer. Math. Soc. 12 (1965), 347-348.

2. P. J. Cohen, The independence of the continuum hypothesis. I, II, Proc. Nat. Acad. Sci. U.S.A. 50 (1963), 1143-1148; 51 (1964), 105-110. 
3. S. Feferman, Some applications of the notions of forcing and generic sets, Fund. Math. 56 (1965), 325-345.

4. S. Feferman and C. Spector, Incompleteness along paths in progressions of theories, J. Symbolic Logic 27 (1962), 383-390.

5. W. Feller, An introduction to probability theory and its applications, Wiley, New York, 1957.

6. R. O. Gandy, Proof of Mostowski's conjecture, Bull. Acad. Polon. Sci. Ser. Math. 8 (1960), 571-575.

7. - On a problem of Kleene, Bull. Amer. Math. Soc. 66 (1960), 501-502.

8. K. Gödel, Consistency proof of the generalized continuum hypothesis, Proc. Nat. Acad. Sci. U.S.A. 25 (1939), 220-224.

9. - The consistency of the axiom of choice and of the generalized continuum hypothesis with the axioms of set theory, 4th printing, Princeton Univ. Press, Princeton, N. J., 1958.

10. P. R. Halmos, Measure theory, Van Nostrand, Princeton, N. J., 1950.

11. S. C. Kleene, On the forms of the predicates in the theory of constructive ordinals (second paper), Amer. J. Math. 77 (1955), 405-428.

12. — Arithmetical predicates and function quantifiers, Trans. Amer. Math. Soc. 79 (1955), 312-340.

13. - Hierarchies of number-theoretic predicates, Bull. Amer. Math. Soc. 61 (1955), 193-213.

14. - Quantification of number-theoretic functions, Compositio Math. 14 (1959), 23-40.

15. G. Kreisel, Set theoretic problems suggested by the notion of potential totality, Proc. Sympos. Infinitistic Methods, Warsaw, 1961, pp. 103-140.

16. - The axiom of choice and the class of hyperarithmetic functions, Indag. Math. 24 (1962), 307-319.

17. G. Kreisel and G. E. Sacks, Metarecursive sets, J. Symbolic Logic 30 (1965), 318-338.

18. S. Kripke, Transfinite recursions on admissible ordinals. I and II (abstracts), J. Symbolic Logic 29 (1964), 161-162.

19. R. A. Platek, Foundations of recursion theory, Ph.D. Thesis, Stanford Univ., Stanford, Calif., 1965.

20. H. Rogers, Theory of recursive functions and effective computability, McGraw-Hill, New York, 1967.

21. J. Rosenthal, Addition of relations in model theory; Truth in all of certain well-founded countable models arising in set theory, Ph.D. Thesis, Massachusetts Institute of Technology, Cambridge, Mass., 1968.

22. G. E. Sảcks, Degrees of unsolvability, Princeton Univ. Press, Princeton, N. J., 1963.

23. - Measure-theoretic uniformity, Bull. Amer. Math. Soc. 73 (1967), 169-174.

24. - Higher recursion theory, Lecture Notes, Massachusetts Institute of Technology, Cambridge, Mass., 1966.

25. D. Scott, Lectures on Boolean-valued models for set theory, Amer. Math. Soc. Summer Institute on Axiomatic Set Theory, UCLA, 1967. Lecture Notes. $\left({ }^{14}\right)$

26. D. Scott and R. Solovay, "Boolean-valued models for set theory" in Axiomatic set theory, Proc. Sympos. Pure Math., vol. 13, Amer. Math. Soc., Providence, R. I. (to appear).

27. J. R. Shoenfield, The problem of predicativity, Essays on the Foundations of Mathematics, Magnes Press, Hebrew Univ., Jerusalem, 1961, pp. 132-139.

28. W. Sierpiński, Les ensembles projectifs et analytiques, Mémor. Sci. Math., no. 112, Gauthier-Villars, Paris, 1950.

${ }^{(14)}$ This reference is a preliminary version of [26]. 
29. R. Solovay, The measure problem, Abstract 65T-62, Notices Amer. Math. Soc. 12 (1965), 217.

30. - The measure problem, (to appear).

31. C. Spector, Recursive well-orderings, J. Symbolic Logic 20 (1955), 151-163.

32. - Measure-theoretic construction of incomparable hyperdegrees, J. Symbolic Logic 23 (1958), 280-288.

33. - Hyperarithmetical quantifiers, Fund. Math. 48 (1959), 312-320.

34. H. Tanaka, Some results in the effective descriptive set theory, Publ. RIMS, Kyoto Univ. Ser A 3 (1967), 11-52.

35. - A basis result for $\Pi_{1}^{1}$-sets of positive measure, Hosei University, 1967.

36. L. Tharp, Set theory, Lecture Notes, Massachusetts Institute of Technology, Cambridge, Mass., 1965.

Massachusetts Institute of Technology,

CAmbridge, Massachusetts 